\title{
Design of High-Gain and Beam Steering Antennas Using a New Planar Folded-Line Metamaterial Structure
}

\author{
Minh Thuy Le, ${ }^{1,2}$ Quoc Cuong Nguyen, ${ }^{1,2}$ and Tan Phu Vuong ${ }^{3}$ \\ ${ }^{1}$ International Research Institute MICA, HUST-CNRS/UMI-2954-Grenoble INP, Hanoi University of Science and Technology, \\ Hanoi 10000, Vietnam \\ ${ }^{2}$ Department of Instrumentation and Industrial Informatics, School of Electrical Engineering, Hanoi University of \\ Science and Technology, Hanoi 10000, Vietnam \\ ${ }^{3}$ IMEP-LAHC Laboratory, UMR 5130 INPG-UJF-CNRS, Grenoble INP, Grenoble Cedex 1, Grenoble 38400, France
}

Correspondence should be addressed to Minh Thuy Le; minh-thuy.le@mica.edu.vn

Received 21 April 2014; Revised 7 July 2014; Accepted 8 July 2014; Published 15 September 2014

Academic Editor: Miguel F. Bataller

Copyright (C) 2014 Minh Thuy Le et al. This is an open access article distributed under the Creative Commons Attribution License, which permits unrestricted use, distribution, and reproduction in any medium, provided the original work is properly cited.

\begin{abstract}
In the last few years, there has been growing interest in employing metamaterials (MTMs) to enhance antenna gain. In this paper we proposed a novel structure of planar folded-line left-handed metamaterial (FL-LHM) and applied it to improve the gain of three $5.8 \mathrm{GHz}$ microstrip antenna types: a circularly polarized patch antenna, an antenna array, and a beam steering antenna. The planar FL-LHM structure was designed based on transmission line analysis. Their scattering parameters were obtained using a numerical model; the negative effective permittivity and permeability were then calculated from these parameters for the assessment of negative refraction index region. The $S_{11}$ and radiation patterns of three fabricated antennas were measured; these results matched well with the simulation. We observed that the gain was increased up to $3 \mathrm{dBi}$ for all the antennas. In addition, we were also able to maintain the circular polarization as well as the steering of the antenna without changing its dimensions.
\end{abstract}

\section{Introduction}

Antenna is an important component that affects the performance of wireless communication systems. Antennas with low profile, low manufacturing costs, and high gain are more desirable for the system. To satisfy the requirements, microstrip antenna is a good candidate for the antenna design. However it is difficult to obtain a high gain using a normal microstrip antenna. To resolve this issue, some traditional technologies for enhanced-gain antenna are used such as reflectors, directors, dielectric lenses, superstrates, or array techniques. In recent years, electromagnetic bandgap (EBG) structures and metamaterials have been demonstrated to enhance the antenna gain. This paper will be focused on the high gain antenna using MTM technique.

Metamaterials denote artificial constructed materials that may not be found in nature. Metamaterials has negative permittivity $(\varepsilon<0)$ and/or negative permeability $(\mu<0)$. The MTM is called double-negative material (DNM) or lefthanded material (LHM) when it has double-negative $\varepsilon$ and $\mu$.
With the same incident wave, the reflected wave through a LHM is in opposition to the reflected wave through a positive permittivity and permeability material. LHM acts like lenses to focus wave in the same direction; thus it is usually placed above an antenna to increase its gain. In general, LHM uses a periodic structure and is modeled as an infinite array of MTM unit-cells. Therefore, in this paper, a single LHM unitcell will be studied instead of the entire array of unit-cells. The dimension of a LHM unit-cell is very small compared to $\lambda_{0}$ where $\lambda_{0}$ is the wavelength in free space at the operating frequency. In this paper, we concentrate on the design of a new LHM and its applications in gain enhancement for lowprofile antennas.

In the context of improving the antenna gain, two types of MTM are usually found: the left-handed materials or doublenegative materials and evanescent materials. LHMs have both negative permittivity and permeability that were mentioned by Veselago in 1968 [1]. His paper introduced the propagation of waves in LHMs that is opposite to the wave vector in the right-handed materials (RHMs); LHMs possess the negative 
TABLE 1: Review of LHM structures.

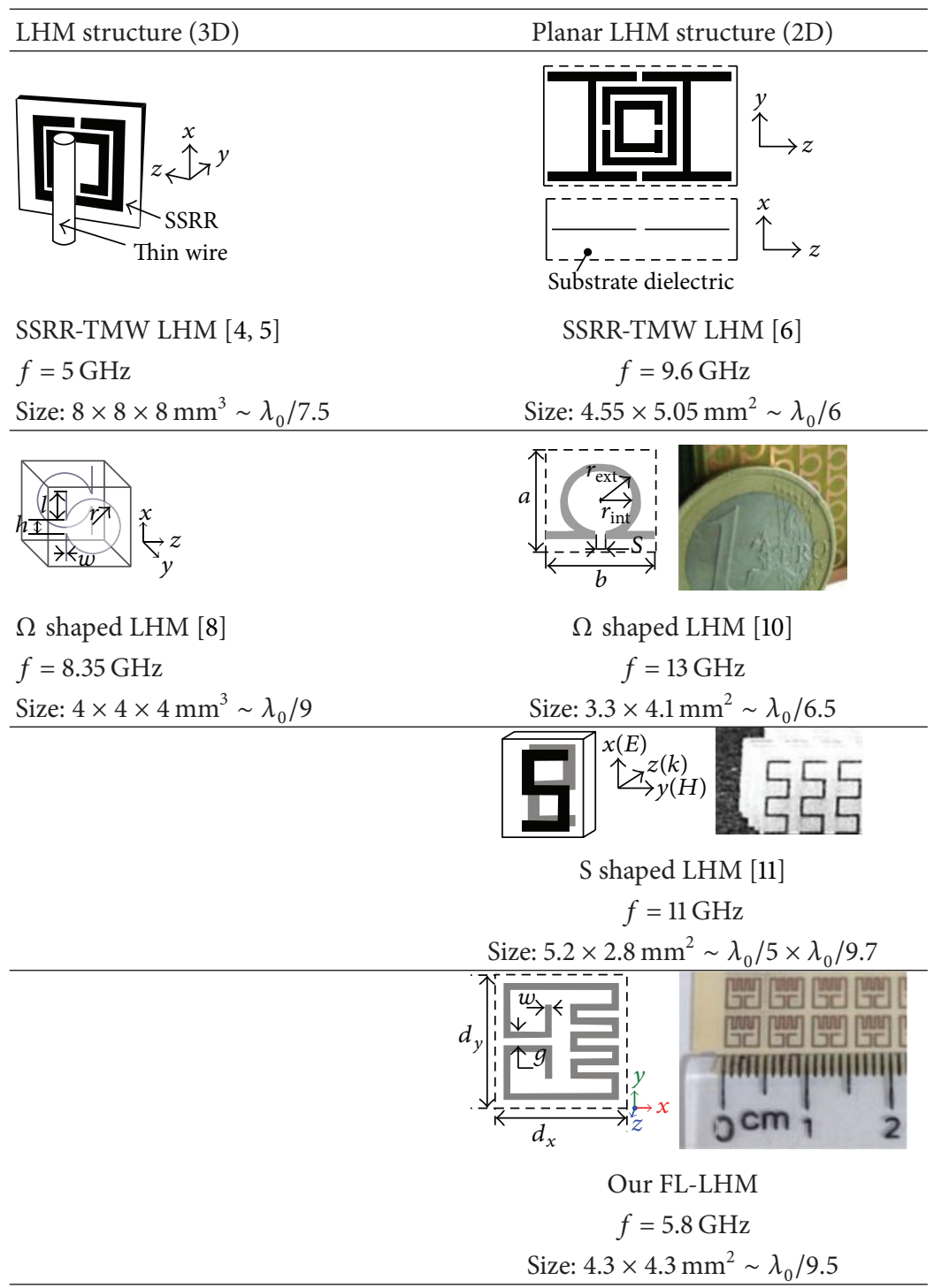

refraction index $(n)$ and their wave vector $k$ is also called the "backward wave." Evanescent materials are the other type of MTMs with single negative $\varepsilon<0$ or $\mu<0$ which were considered by Prendry and his colleagues thirty years later. He found out that thin metallic wire lattices (TMWs) had effectively negative permittivity in [2] and split ring resonators (SRRs) had effectively negative permeability in [3] in specified frequency bands. The first practical LHM unit-cell structure was proposed in $[4,5]$ by Smith and his colleagues based on SRR of Prendry in [3]. It is constituted of TMW and SRR which have dimension in 3D of $d=\lambda_{0} / 7.5$ $\left(d_{x} \times d_{y} \times d_{z}\right)$. Ziolkowski then successfully investigated and realized some slabs of planar LHM structures that comprised a substrate Duroid $5880\left(h_{\text {sub }}=0.8 \mathrm{~mm}\right)$ with embedded strip line operating at the $X$ band [6]. This one is more compact than the first LHM structure in $3 \mathrm{D}$ and suitable for low-profile antenna application. The dimension of these planar LHM unit-cells in 2D is around $d=\lambda_{0} / 6\left(d_{x} \times d_{y} \times h_{\text {sub }}\right)$. The other LHM structure, the " $\Omega$ shaped," was first suggested in 1992 by
Saadoun and Engheta [7]. In 1997, Simovski et al. presented " $\Omega$ " shaped LHM unit-cell in $3 \mathrm{D}$ with the dimension of $d=$ $\lambda_{0} / 9\left(d_{x} \times d_{y} \times d_{z}\right)$, application for antenna gain enhancement in [8]. This LHM unit-cell is smaller than the LHM unit-cell of Prendry and Smith. At the same time, they also designed the " $\Omega$ " shaped LHM unit-cell in $2 \mathrm{D}$ in [9]; then the " $\Omega$ " shaped LHM unit-cell in 2D was fabricated in 2008 [10] with the dimension of $d=\lambda_{0} / 6.5\left(d_{x} \times d_{y} \times h_{\text {sub }}\right)$ using Roger Duroid substrate. The new planar "S" shaped LHM unit-cell was investigated by Chen et al. in [11] with the dimension of $\lambda / 5 \times \lambda / 9.7 \times h_{\text {sub }}$, but this LHM could not be smaller than $\lambda_{0} / 6,5$ (see Table 1 ).

In addition, the negative effective permittivity, permeability, and refraction index can be extracted from average $H$ field and E-field of each LHM unit-cell [6, 12-15] or from their reflection and transmission coefficient parameters [1618]. These methods have been researched and validated by many researchers, especially matched results between the simulation and the experimental $S$ parameters which have 
been demonstrated in $[16,18-20]$. For this reason, our new planar "folded-line" LHM (FL-LHM) unit-cell structure will retrieve their effective $\varepsilon$ and $\mu$ from $S$ parameters based on the numerical LHM unit-cell model. This FL-LHM unit-cell has a smallest dimension of $\lambda_{0} / 9.5$ compared with the published unit-cell structures which are listed in Table 1. In Section 2, we show the methodology to design and to obtain this FLLHM at defined operating frequency.

The design of novel high gain and beam steering antennas using FL-LHM substrate will be presented more in detail in Section 3. When a LHM substrate covers a reference antenna, it enhances the gain of that antenna and also maintains its performance. This performance can be circular polarization or beam steering. This one is a major advantage of LHM substrate which will be presented in Section 2. As we have reported in our previous work [21,22], the operating frequency of FL-LHM as well as FL-LHM antenna was defined at $5.8 \mathrm{GHz}$ in order to satisfy the operation-range requirement for reader antenna of electronic-toll-collection (ETC) free-flow system application on the highway in Europe. The ETC free-flow system allows automatic fee payments of vehicles without stopping on the highway. It is composed of a reader and transponders (badges) where the reader is fixed on a gantry of the road and the badge is mounted on a vehicle $[22,23]$. Each badge stores all information of each vehicle, such as the class, the owner of vehicle, his address, and his bank account. Reader detects and then communicates with badge to collect all vehicle information when a vehicle enters its operating zone. The fee is offered and then paid based on this collected information between reader and badge. Physical layer of the equipment (the reader and the badge) uses the microwave communication at the spectrum of $5.795 \mathrm{GHz}-5.815 \mathrm{GHz}$ or $5.875 \mathrm{GHz}-5.905 \mathrm{GHz}$ according to European dedicated short-range communication (DSRC) standard [24, 25]. In this DSRC system, an antenna with higher gain gives a longer distance of communication and hence vehicles can be allowed to pass faster. In addition, an antenna only covers a lane: if a beam steering antenna is used, it could cover multilanes; therefore the price and the size of highway equipment in ETC free-flow system will be reduced. The high gain, low profile, and multibeams are always the requirements for designing of antenna at $5.8 \mathrm{GHz}$ in this system.

\section{Theory and Design of New Planar FL-LHM}

FL-LHM substrate is created by periodic arrays of $N_{x} \times N_{y}$ FL-LHM unit-cells in $x$ and $y$ directions. Hence, to design a LHM substrate, we primarily focus on the design of a new planar FL-LHM unit-cell.

2.1. Transmission Line Analysis. LHM substrate is created from periodic LHM unit-cells. Each planar unit-cell consists of two conductor faces etched on a substrate. The shapes of these two conductor faces are the same. Thus, a LHM unitcell is described by the equivalent circuit using transmission line method as shown in Figure 1.

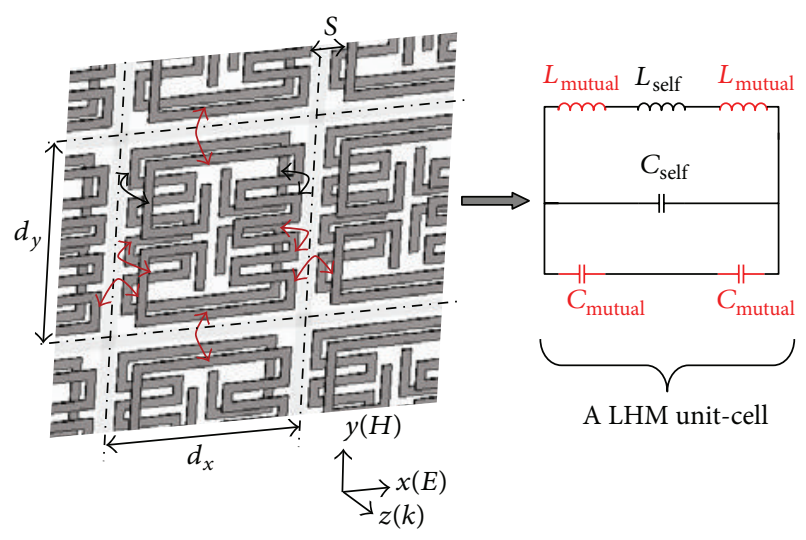

- Self-impedance

FIGURE 1: LHM substrate is created by periodic arrays of LHM unitcells (left) and the equivalent circuit of a unit-cell (right); excitation waves (from the reference antenna) are coming to LHM substrate in $z$ direction.

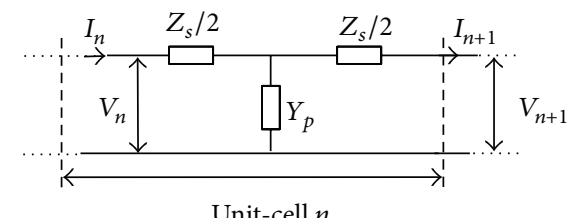

Unit-cell $n$

FIGURE 2: LHM unit-cell is described by symmetrical circuit model.

According to this circuit, the resonant frequency of unitcell can be estimated using the formula $f=1 / 2 \pi \sqrt{L_{t} C_{t}}$, where $L_{t}$ and $C_{t}$ denote total inductance and total capacitance of unit-cell, respectively:

$$
\begin{aligned}
& L_{t}=L_{\text {self }}+2 L_{\text {mutual }}, \\
& C_{t}=C_{\text {self }}+\frac{C_{\text {mutual }}}{2} .
\end{aligned}
$$

The gap $s$ between two conductors of two adjacent unit-cells determines their mutual coupling level. The closer the unitcells are, the larger the current magnitude is; thus the resonant frequency will be increased; refer to (1). We found that these components define resonant frequency like effective permittivity and permeability. For easier understanding and designing, each unit-cell is represented by a symmetrical circuit model as in Figure 2 according to $[14,26]$, where the total inductance $L_{t}$ has been split into series $\left(L_{s}\right)$ and parallel $\left(L_{p}\right)$ components, similarly for the total capacitance $C_{t}$.

$L_{s}$ depends on the total length of conductor line $l$ and its value is dominant in series impedance $\left(Z_{s}\right)$. On the other hand, $C_{p}$ depends on area of parallel surface between two conductor faces; its value is dominant in shunt admittance $\left(Y_{p}\right)$ and depends on the "common" parallel area. As consequence, we can change the total length of 


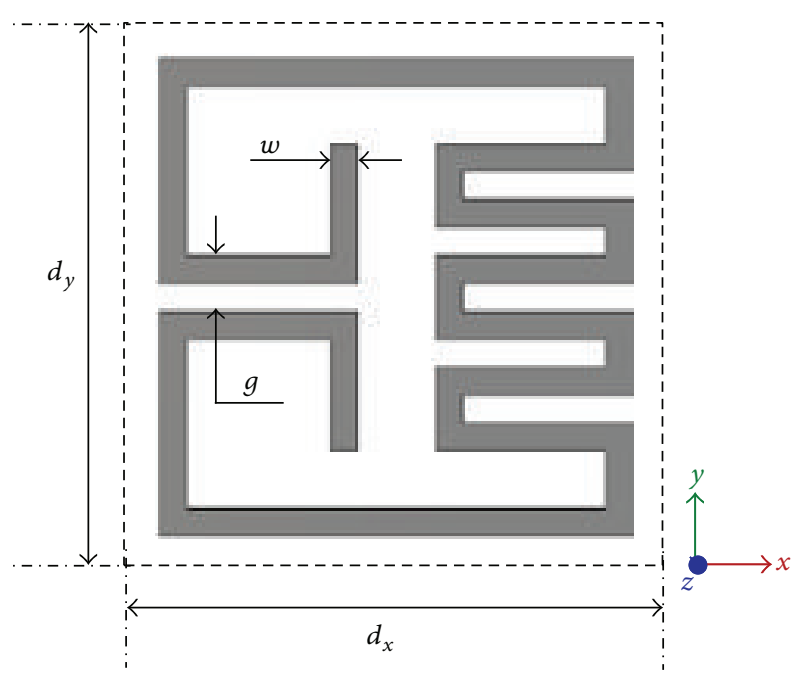

(a)

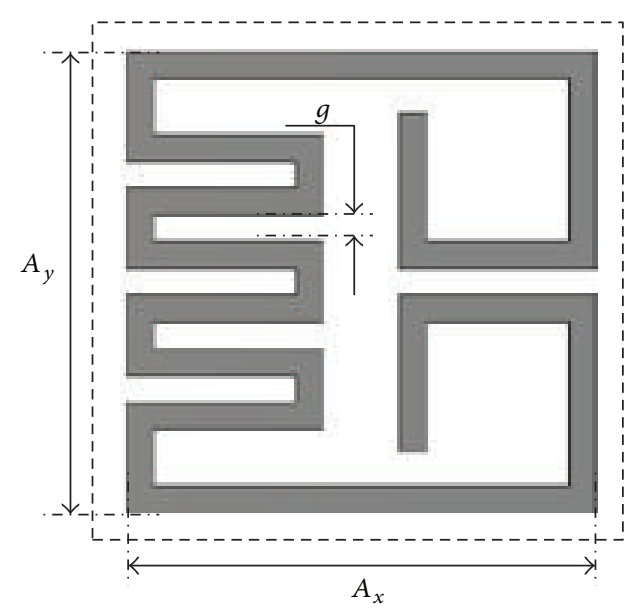

(c)

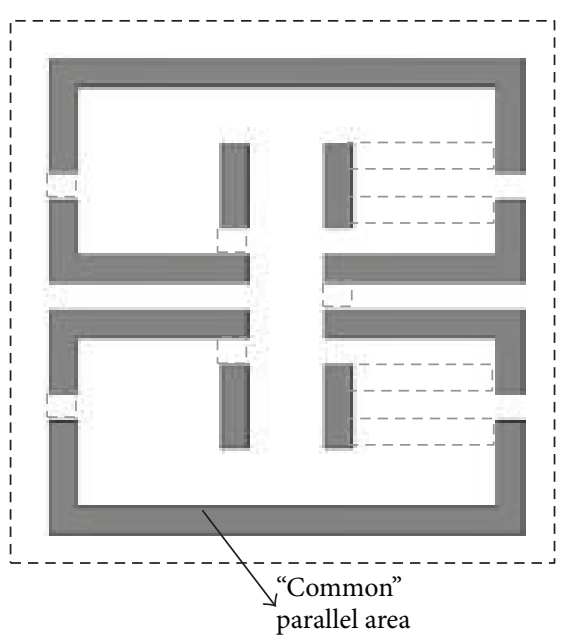

(b)

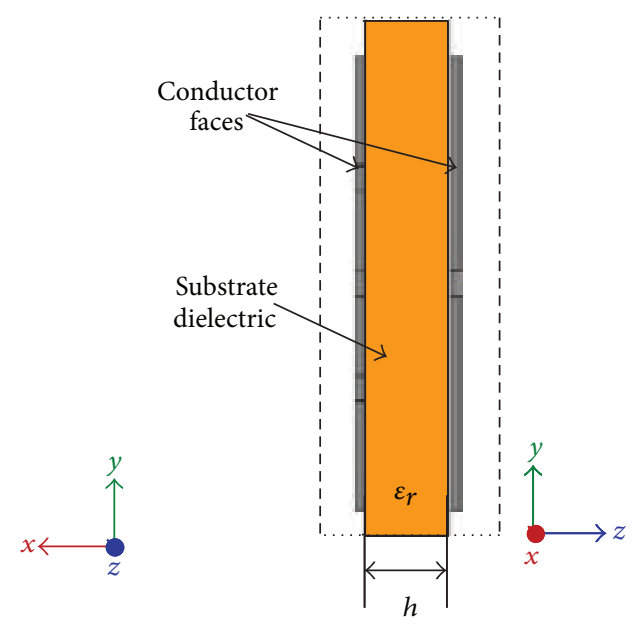

(d)

Figure 3: New FL-LHM unit-cell structure for resonant frequency at 5.8 GHz: (a) front view of FL-LHM unit-cell; (b) "common" parallel area of unit-cell; (c) back view; (d) side view. The details of parameters are summarized in Table 2.

TABLe 2: Parameters of new FL-LHM in Figures 1 and 3.

\begin{tabular}{lc}
\hline Symbol & Value \\
\hline$w$ & $0.25(\mathrm{~mm})$ \\
$l$ & $28.25(\mathrm{~mm})$ \\
$g$ & $0.25(\mathrm{~mm})$ \\
$s$ & $2(\mathrm{~mm})$ (gap between two folded lines) \\
$A_{x}$ & $4.3(\mathrm{~mm})$ \\
$A_{y}$ & $4.3(\mathrm{~mm})$ \\
$d_{x}$ & $5.3(\mathrm{~mm})($ dimension of a unit-cell in $x$ direction) \\
$d_{y}$ & $5.3(\mathrm{~mm})$ (dimension of a unit-cell in $y$ direction) \\
$\varepsilon_{r}$ & 3.55 permittivity of substrate $(3.55+j 0.0027)$ \\
$\mu_{r}$ & Permeability of substrate \\
\hline
\end{tabular}

line $(l)$ or the "common" parallel area to achieve desired resonant frequency. This means the higher the $l$ or "common" parallel area is, the lower the resonant frequency is. The series impedance and shunt admittance of a unit-cell can be obtained from

$$
\begin{gathered}
Z_{s}=-j \omega L_{s}-\frac{1}{j \omega C_{s}}, \\
Y_{P}=-j \omega C_{p}-\frac{1}{j \omega L_{p}} .
\end{gathered}
$$

The effective permittivity and permeability of unit-cell in this model in Figure 2 can be calculated using the Bloch theorem. We start from the relation of the current and the voltage that passes thought a unit-cell as the following equation:

$$
\begin{gathered}
I_{n+1}=I_{n} e^{j \beta}, \\
V_{n+1}=V_{n} e^{j \beta},
\end{gathered}
$$


where $\beta$ is the phase crossing through unit-cell $n$ :

$$
\beta=k p,
$$

where $k$ is the wave vector in the unit-cell and $p$ is the dimension of the periodic unit-cell. As in Figure 1, $p=d_{x}=$ $d_{y}=d$.

Involving the spatial dispersion in these effective parameters, according to $[14,26]$, the effective permittivity and permeability of a unit-cell can be calculated from the following equations:

$$
\begin{gathered}
\mu_{\mathrm{eff}}=\frac{\omega L_{s}-\left(1 / \omega C_{s}\right)}{2 \omega p \beta \tan (\beta / 2)}, \\
\varepsilon_{\mathrm{eff}}=\frac{2 \beta \tan (\beta / 2)}{\left(\omega L_{s}-\left(1 / \omega C_{s}\right)\right) \omega p} .
\end{gathered}
$$

The phase crossing through one unit-cell $\beta$ in (4) can be now obtained by (6) with the boundary condition in (7):

$$
\begin{gathered}
\sin ^{2}\left(\frac{\beta}{2}\right)=\frac{Z_{s^{\prime}} Y_{p^{\prime}}}{4}, \\
0 \leq \frac{Z_{s^{\prime}} Y_{p^{\prime}}}{4} \leq 1 \Longleftrightarrow 0 \leq Z_{s^{\prime}} Y_{p^{\prime}} \leq 4,
\end{gathered}
$$

where $Z_{s^{\prime}}, Y_{p^{\prime}}$ are real numbers; they can be negative or positive depending on the values of $L_{s}, C_{s}$ and $L_{p}, C_{p}$ as follows:

$$
\begin{aligned}
& Z_{s^{\prime}}=\omega L_{s}-\frac{1}{\omega C_{s}}, \\
& Y_{p^{\prime}}=\omega C_{p}-\frac{1}{\omega L_{p}} .
\end{aligned}
$$

The wave impedance of LHM unit-cell is

$$
Z=\frac{V_{n}}{I_{n}}=\frac{1}{2} \frac{Z_{s^{\prime}}}{\tan (\beta / 2)} .
$$

From (5) we can summarize that a LHM unit-cell can be obtained by choosing suitable values of $L_{s}, C_{s}$ and $L_{p}, C_{p}$ under the condition in (7) combined with (10):

$$
\omega L_{s}<\frac{1}{\omega C_{s}} .
$$

As presented, the total length of the conductor line increases, while the resonant frequency increases. This way, we can tune the FL-LHM to any operating frequency. For convenience, at the frequency of $5.8 \mathrm{GHz}$ of LHM unit-cell $5.8 \times 10^{9}=1 / 2 \pi \sqrt{L_{t} C_{t}}$, we suppose that $L_{t}$ and $C_{t}$ are defined as $L_{s}=32.73 \mathrm{nH}, C_{s}=0.018 \mathrm{pF}$, and $C_{p}=0.95 \mathrm{pF}$ with $\omega C_{p}<1 / \omega L_{p}$. As in Figure 3, the investigated FL-LHM unitcell consists of two conductor lines etched on Roger 4003 substrate and has the following dimensions.

(i) Each conductor face is created by a line with the width of $w=0.25 \mathrm{~mm}$ and the total length of $l=$
$28.25 \mathrm{~mm}$ (around $\lambda_{0} / 2$ ) to satisfy conditions (7) and (10) above to have resonant frequency at $5.8 \mathrm{GHz}$. This line is folded in one unit-cell with dimensions of $4.3 \times$ $4.3 \mathrm{~mm}^{2}\left(\lambda_{0} / 9.5\right)$ by using meander line structure in $y$ direction as in Figures 3(a) and 3(c) to reduce the dimension.

(ii) The separation between two unit-cells is of $s=2 \mathrm{~mm}$.

(iii) Two conductor lines are maintained parallel to each other by the substrate dielectric Roger 4003 that has thickness of $h_{\text {sub }}=0.8 \mathrm{~mm}$, permittivity of $\varepsilon_{r}=3.5$, permeability of $\mu_{r}=1$, and loss tangent of $\tan \delta=$ 0.0027 . The "common" parallel area between these two conductors is defined as in Figure 3(b).

The novel FL-LHM has both negative effective permittivity and permeability which are denoted by $\varepsilon_{\text {eff }}$ and $\mu_{\text {eff }}$. Their real parts are negative while the imaginary parts are nearly equal to zero at the operating frequency of $5.8 \mathrm{GHz}$.

2.2. Numerical FL-LHM Model. A quantity of $L$ and $C$ values can be calculated to have a desired FL-LHM using the transmission line analysis in Section 2.1. However, the mutual inductance and capacitance as well as fringing effect are difficult to evaluate. In addition, this quantification will be more complicated when the incident wave varies. Therefore, a numerical model in Figure 4 is created to simplify the design of a FL-LHM unit-cell and the evaluation of their effective permittivity and permeability likewise.

Figure 4(a) illustrates a FL-LHM antenna model; the FLLHM substrate is excited by a reference antenna (RA) which could be any type of antennas. In this case, the pattern of $\mathrm{RA}$ is equivalent to an incident plane wave at the direction varying from $-\theta$ to $\theta$. The released wave from a FL-LHM is propagating in $+z$ direction. These waves consist of the forward wave (solid red line) and the wave reflected at the back FL-LHM (dotted blue line). Both waves have the same phase, so that antenna gain is improved.

From this FL-LHM antenna model, we create FL-LHM unit-cell modeling as in Figure 4(b). The FL-LHM unit-cell is excited by an incidence wave in $z$ direction. To cover all the types of RA, the excitation of FL-LHM unit-cell is modeled by a plane wave incident in direction of theta $\left(-90^{\circ}<\theta<90^{\circ}\right)$. The released wave from a FL-LHM is propagating in $+z$ direction. Hence, $z_{\max }$ is set to perfectmatched layer (PML) (open boundary). Due to a geometrical and electrical symmetry of each unit-cell in Figure 2, the sidewall of each unit-cell model can be replaced by periodic boundary conditions. Particularly, the boundaries $x=x_{\min }$ and $x=x_{\max }$ and $y=y_{\min }$ and $y=y_{\max }$ are set to be periodic boundaries. From this model, the field distribution and the reflection-transmission coefficients of a FL-LHM unit-cell under a normally incident plane wave at any angle $\theta$ are calculated as in Figures 5 and 6 using commercial electromagnetic software CST Microwave Studio 2012. The effective $\varepsilon_{\text {eff }}, \mu_{\text {eff }}$ and refractive index $n$ of FL-LHM can be extracted from $S$ parameters; this method has been validated and demonstrated a good agreement between simulation 


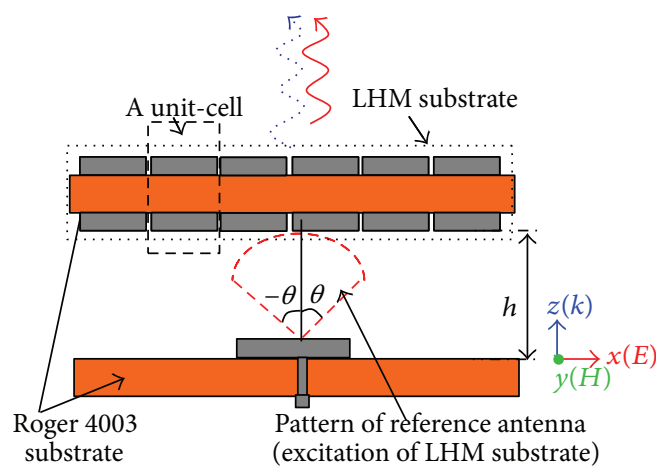

(a)

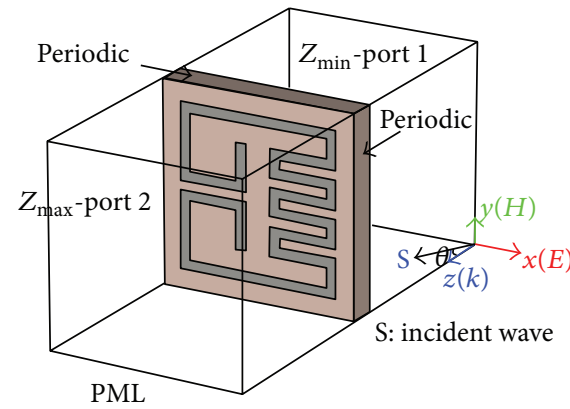

(b)

Figure 4: (a) FL-LHM antenna model which improves the antenna gain by FL-LHM substrate. The reference antenna makes excitation for FL-LHM substrate. The solid red line represents the forward wave and the dotted blue line indicates the wave reflected at the back FL-LHM. (b) Numerical FL-LHM unit-cell model for simulation.

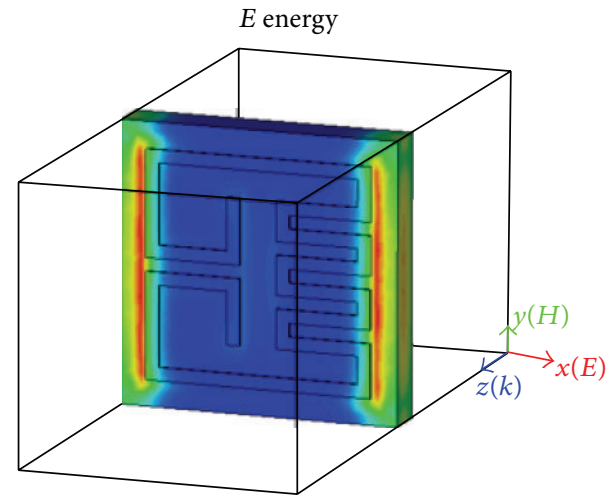

(a)
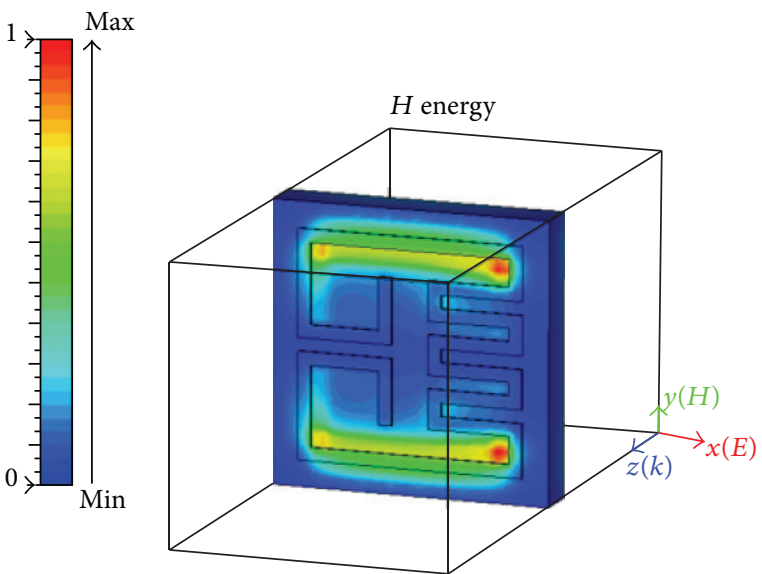

(b)

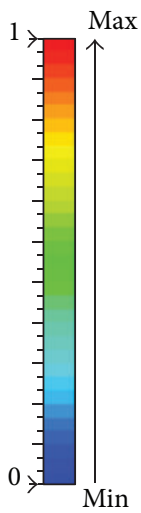

FIGURE 5: Electric and magnetic field distribution in FL-LHM unit-cell.

and measurement in [16-18]. The retrieval of these effective parameters will be shown in the next section.

\subsection{Retrieval of Effective Permittivity and Permeability of the} New FL-LHM from S Parameters. Considering our numerical FL-LHM model, the wave propagation through the FL-LHM is shown as in Figure 7.

As we presented in Section 2.2, the reflection and transmission coefficients $\left(S_{11}\right.$ and $\left.S_{21}\right)$ of the FL-LHM unit-cell that are created from Section 2.1, according to $[9,16]$, are given by these equations:

$$
\begin{aligned}
& S_{11}=\frac{b_{1}}{a_{1}}=\frac{\left(1-T^{2}\right) R}{1-R^{2} T^{2}}, \\
& S_{21}=\frac{a_{2}}{a_{1}}=\frac{\left(1-R^{2}\right) T}{1-R^{2} T^{2}},
\end{aligned}
$$

where $R$ is the reflection coefficient of an incident wave on the interface between free space and FL-LHM, whereas $T$ is the transmission term through the FL-LHM slab:

$$
\begin{gathered}
R=\frac{Z-Z_{0}}{Z+Z_{0}}=\frac{z-1}{z+1}, \\
T=e^{-j k_{0} n d},
\end{gathered}
$$

where $Z_{0}, k_{0}$ are wave impedance and wave number in free space, respectively. The normalized wave impedance $z=$ $Z / Z_{0}$ and refractive index $n$ of the FL-LHM can be expressed in terms of scattering parameters as

$$
\begin{gathered}
z= \pm \sqrt{\frac{\left(1+S_{11}\right)^{2}-S_{21}^{2}}{\left(1-S_{11}\right)^{2}-S_{21}^{2}}}, \\
n=-\frac{1}{k_{0} d}\left\{\left[[\ln (T)]^{\prime \prime}+2 m \pi\right]-j[\ln (R)]^{\prime}\right\},
\end{gathered}
$$




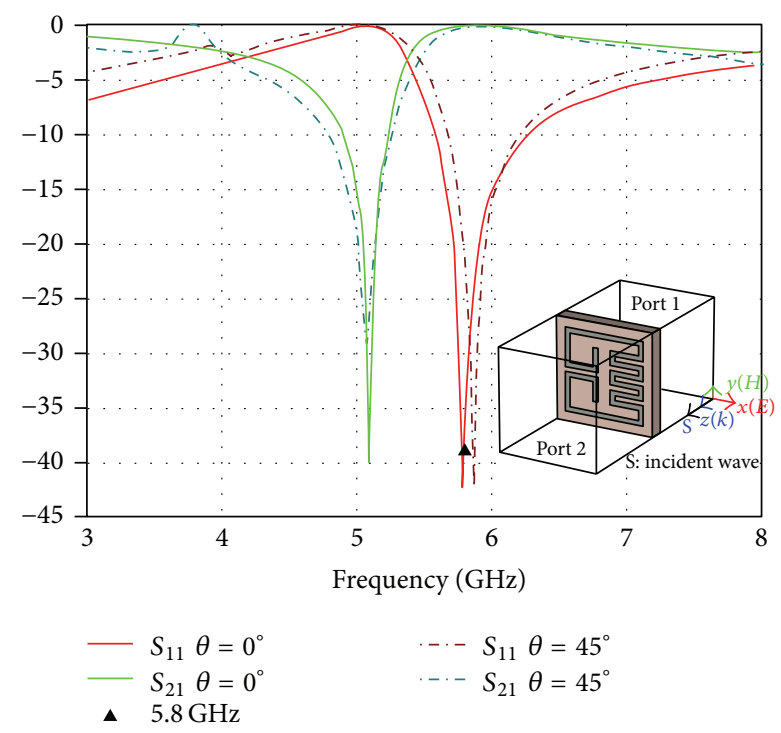

FIGURE 6: Reflection and transmission coefficient in dB of FL-LHM unit-cell under an incident wave at angle of $0^{\circ}$ and $45^{\circ}$.

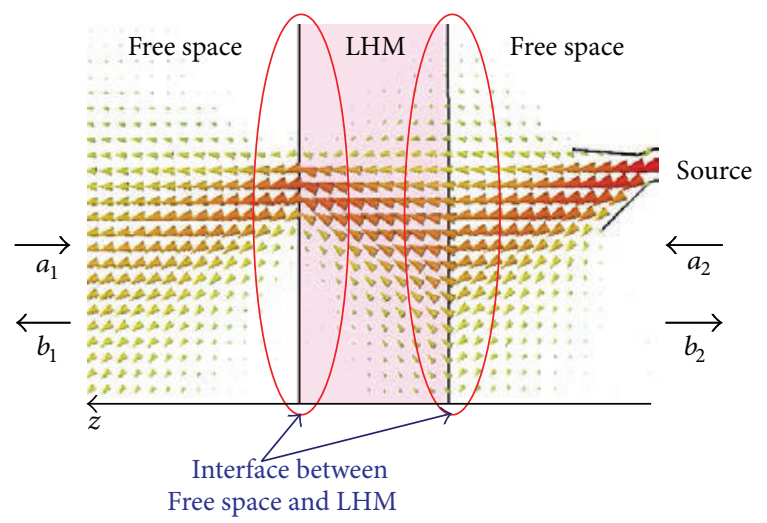

FIGURE 7: Wave propagation of an infinite slab FL-LHM in free space.

where $m$ is an integer related to the branch index of $n^{\prime}$ (principal value of $m=0$ ) and the transmission term as a function of scattering parameters is given by [14]

$$
T=\frac{1-S_{11}^{2}+S_{21}^{2}}{2 S_{21}} \pm j \sqrt{1-\left(\frac{1-S_{11}^{2}+S_{21}^{2}}{2 S_{21}}\right)^{2}} .
$$

The effective permittivity and permeability of the FLLHM are directly calculated from the refractive index $n$ and normalized impedance $z$ :

$$
\varepsilon_{\mathrm{eff}}=\frac{n}{z} ; \quad \mu_{\mathrm{eff}}=n z
$$

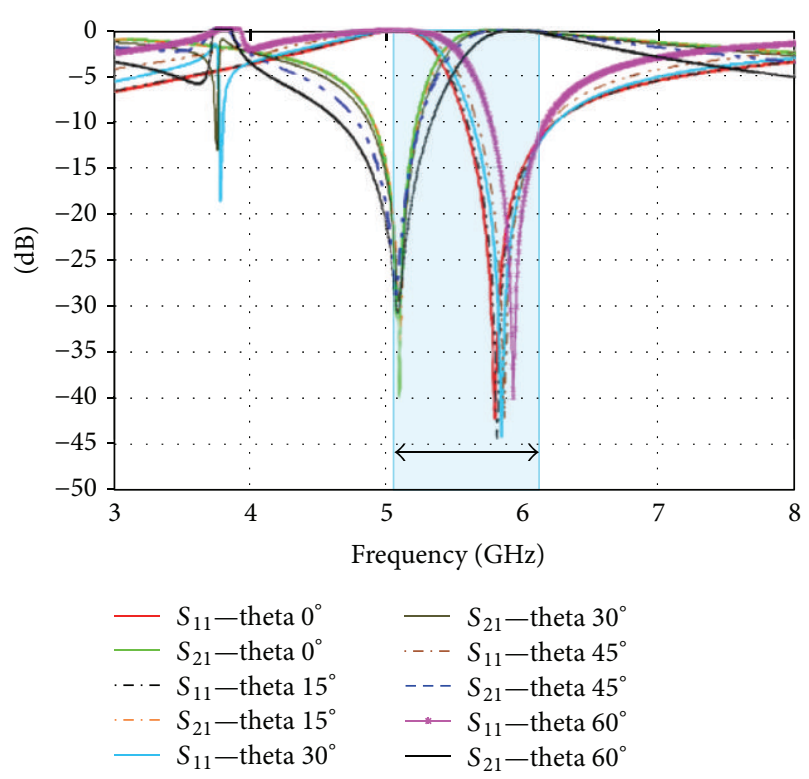

Figure 8: Reflection and transmission coefficients of FL-LHM unitcell under incident wave at any direction from $0^{\circ}$ to $90^{\circ}$.

The retrieval of effective permittivity and permeability of any metamaterial from the scattering parameters is a sufficiently accurate method which allows characterizing a FLLHM. Since the FL-LHM is not homogeneous, the improvement based on the determination of two effective boundaries [19] needs to be determined to increase the accuracy. Besides, the measurement/simulation noise of $S$ parameters influent on the effective impedance is also considered. This method gives us a theoretical validation of the effective permittivity and permeability of the FL-LHM substrate and its dimension from the $S$ parameters results. Because of the periodic structure, we only consider the varying incident angle from $0^{\circ}$ to $90^{\circ}$; the results are repeated with $-90^{\circ}<\theta<0^{\circ}$. S parameters at any angle are shown in Figure 8. We found that $S$ parameters are nearly stable when theta squints from $0^{\circ}$ to less than $30^{\circ}$; only one resonant frequency at $5.8 \mathrm{GHz}$ is obtained. Varying theta in the range from $30^{\circ}$ to $50^{\circ}$, these $S$ values are changed, the resonant frequency is increased above $5.8 \mathrm{GHz}$, and the second resonant frequency at $3.8 \mathrm{GHz}$ has been added. The resonant frequency is shifted as the theta increases. This gives limited condition for RA pattern in FLLHM antenna, especially in the case of a steering RA.

From the $S$ parameters, obtained based on numerical FLLHM model, combined with the retrieval method according to (11)-(15), the effective parameters of our new FL-LHM are presented in Figures 9-12. Both desired negative $\varepsilon_{\text {eff }}$ and $\mu_{\text {eff }}$ are obtained in the range of $5.5-6.2 \mathrm{GHz}$ (LHM bandwidth) according to Figures 10 and 11 while the effective refraction index is negative in the range of 5.1-6.2 GHz (MTM bandwidth). At this LHM bandwidth, their real parts (solid lines) are negative while imaginary parts (dotted lines) are nearly equal to zero which shows that this FL-LHM works well with the low loss at this range, especially in the range of $-45^{\circ} \leq \theta \leq 45^{\circ}$ as in Figures 9 and 10. 


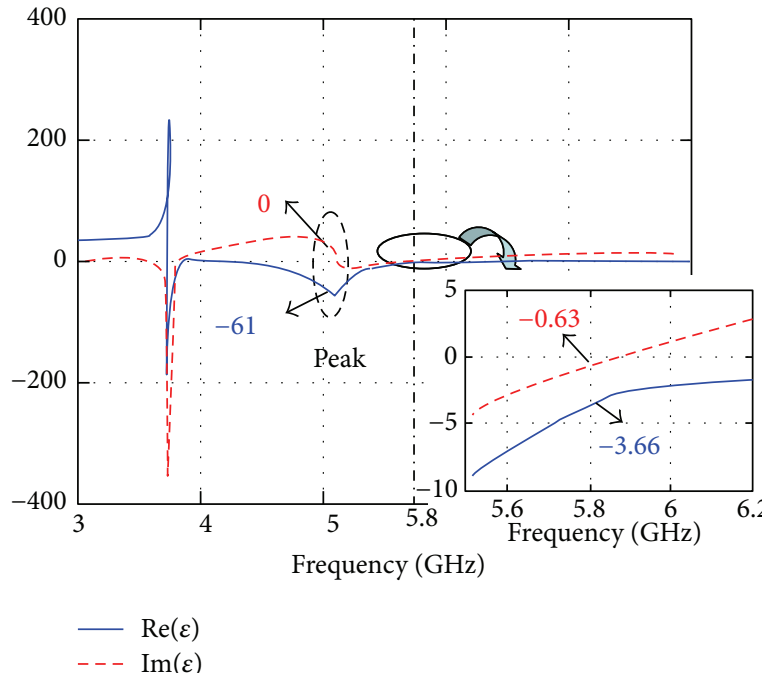

(a)

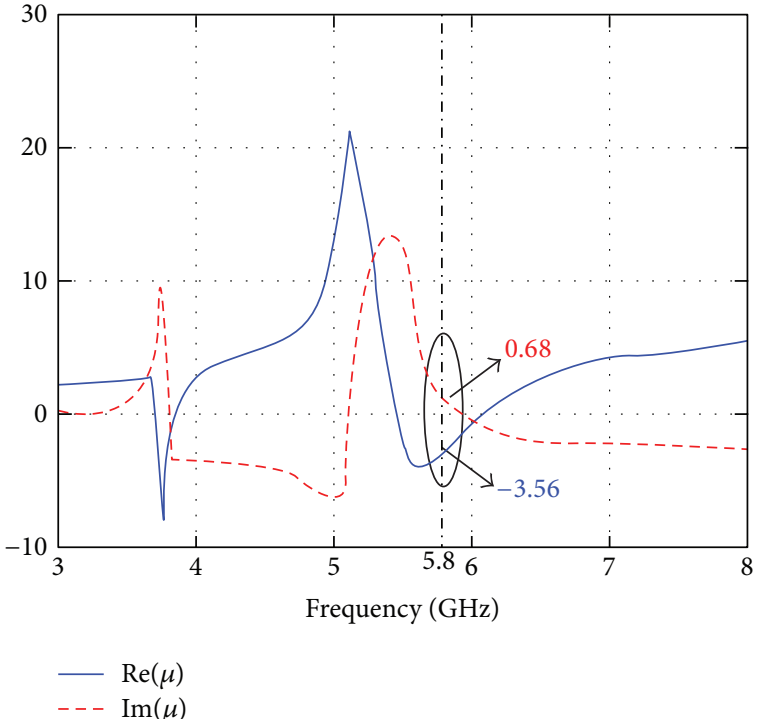

(b)

Figure 9: Effective permittivity (a) and permeability (b) of FL-LHM unit-cell under an incident wave of $45^{\circ}$ extracted from $S$ parameters correspondent.

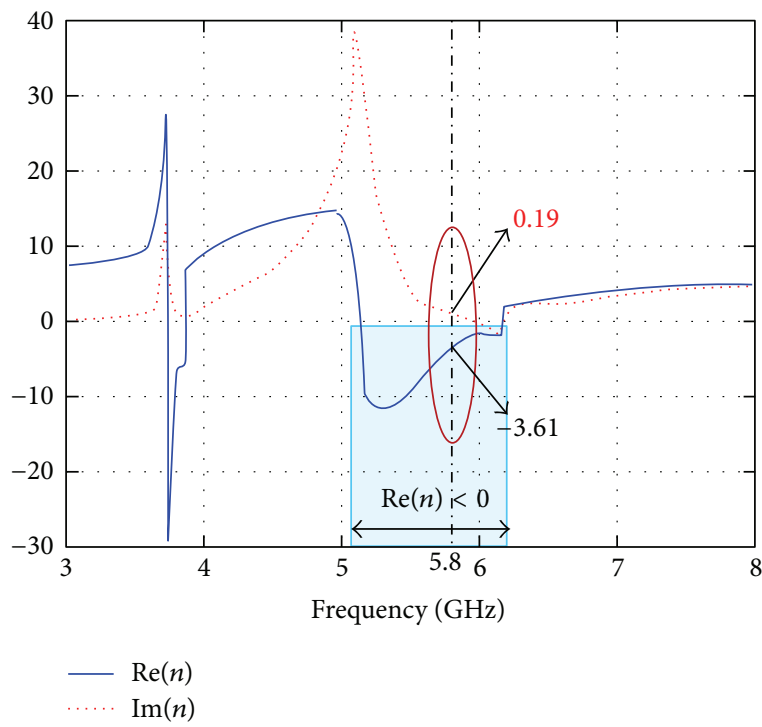

(a)

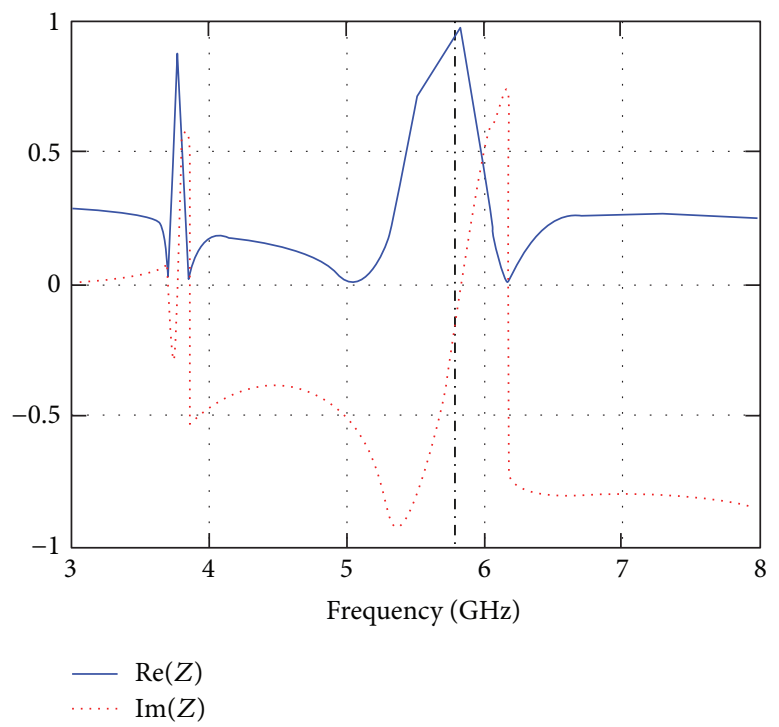

(b)

FIGURE 10: Effective refraction index (a) and normalized impedance (b) of FL-LHM unit-cell under an incident wave of $45^{\circ}$ extracted correspondent.

\section{FL-LHM in Enhanced-Gain for 5.8 Patch and Beam Steering Antenna}

In general, the gain of a microstrip patch antenna is around 6-7 $\mathrm{dBi}$. The gain can be increased by using antenna arrays (adding dimensions in $x, y$ directions), metamaterial technology (only changing dimension in $z$ direction), or both of them. The LHM antenna structure is presented in Figure 4(a); it consists of a RA and a FL-LHM substrate to increase the overall gain. Interestingly, this increasing gain is in good agreement with any type of RA such as the circular polarization antenna or beam steering antenna. For experimental verification of the enhanced-gain effect of FL-LHM substrate, we have realized three types of RA: the patch antenna, the antenna arrays of four patches, and the beam steering patch antenna. The Vector Network Analyzer 8510C is used for $S_{11}$ 


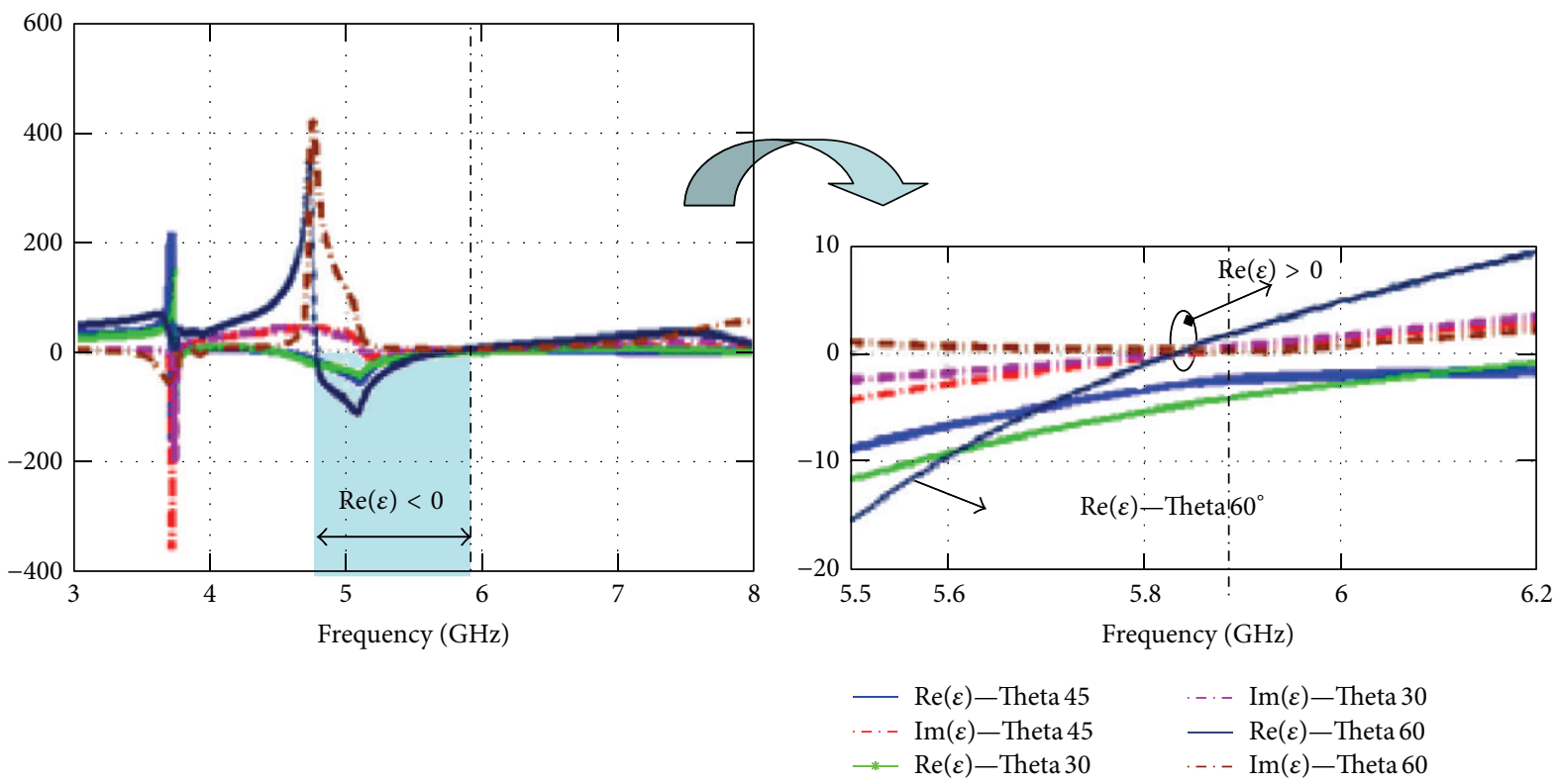

FIGURE 11: Effective permittivity values are obtained with varying incident wave.

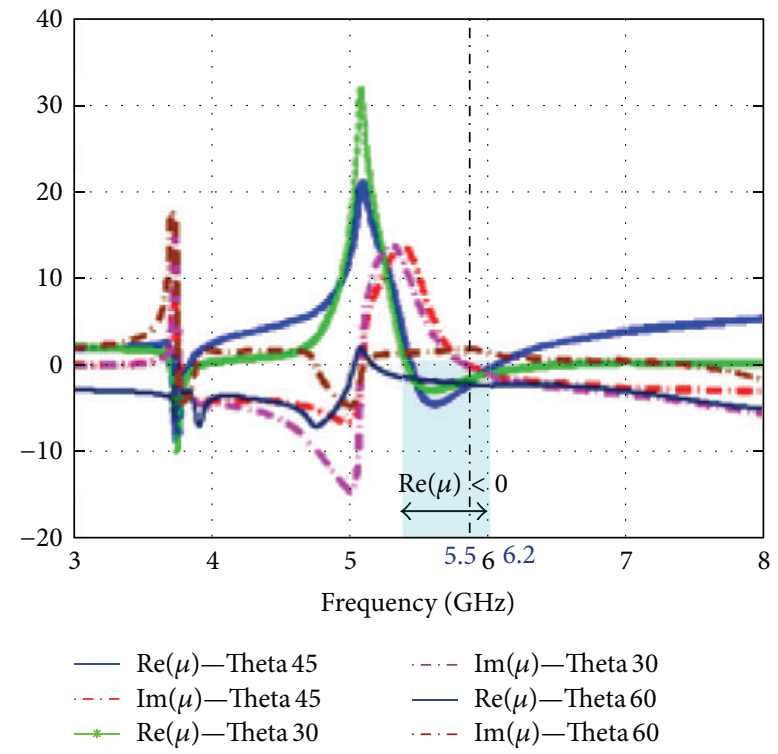

(a)

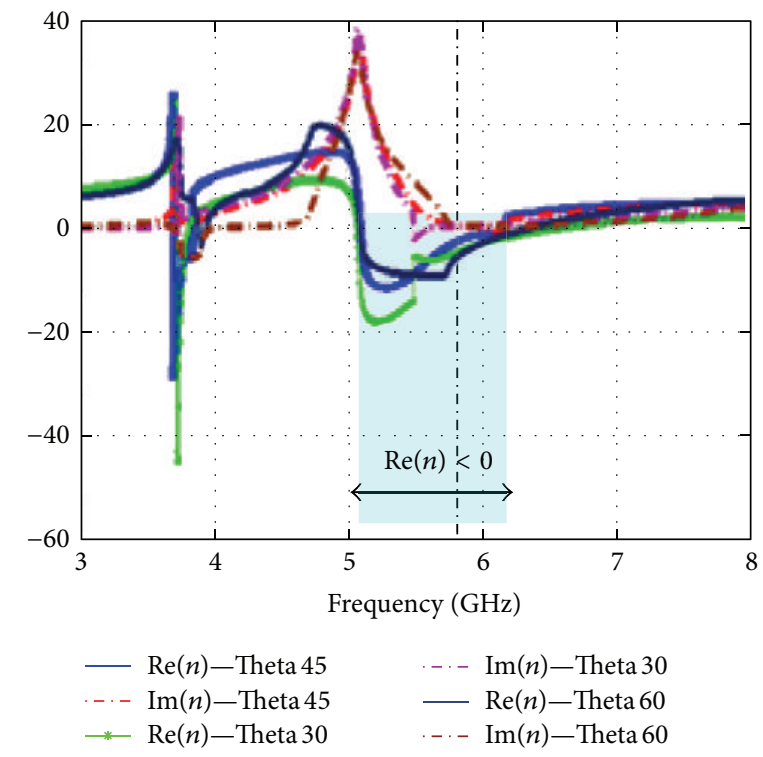

(b)

FIGURE 12: Effective permeability (a) and refraction index (b) values are obtained with varying incident wave.

measurement. The measurement of radiation pattern antenna is performed using the anechoic chamber in our laboratory.

The size of FL-LHM substrate is an important parameter that needs to be defined. According to analysis in Section 2, especially in Figure 4(a), when a RA is covered by a suitable FL-LHM substrate at the height $h=\lambda_{0} / 2$, the RA gain will be improved and the LHM antenna is always well matched at operating frequency. In general, dimensions of FL-LHM substrate $\left(L_{x}, L_{y}\right)$ are proportional to the angular width of
RA and the air-gap height of $h$ between RA and FL-LHM substrate. In addition, suitable FL-LHM substrate dimensions are optimized depending on the dimension of RA as well as the application systems.

3.1. Circularly Polarized Patch Antenna Gain Enhancement. A circularly polarized rectangular patch antenna with dimensions of $46 \times 46 \times 0.8 \mathrm{~mm}$ has been created. This RA uses 


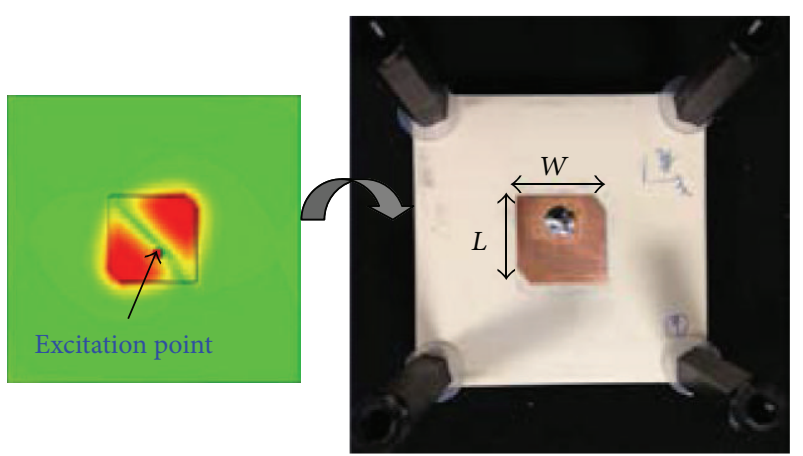

(a)

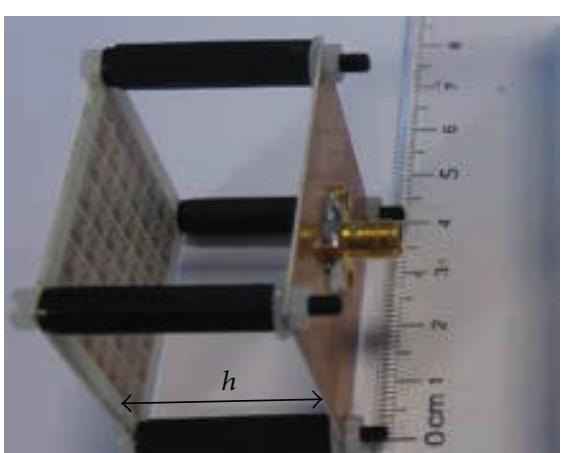

(b)

Figure 13: Prototype of FL-LHM antenna: (a) circularly polarized patch reference antenna; (b) prototype of FL-LHM antenna.

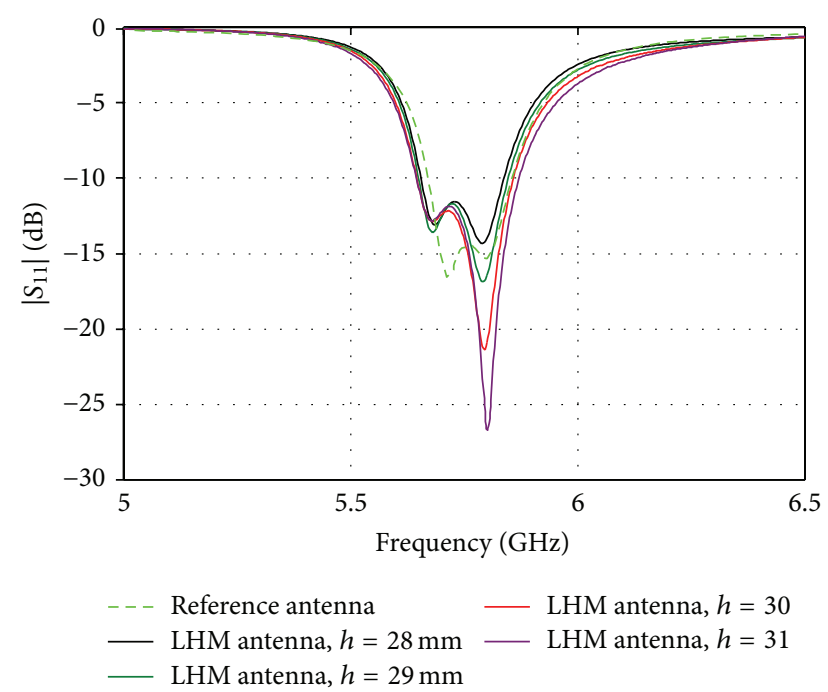

FIGURE 14: Reflection coefficients of antenna with varying air-gap height.

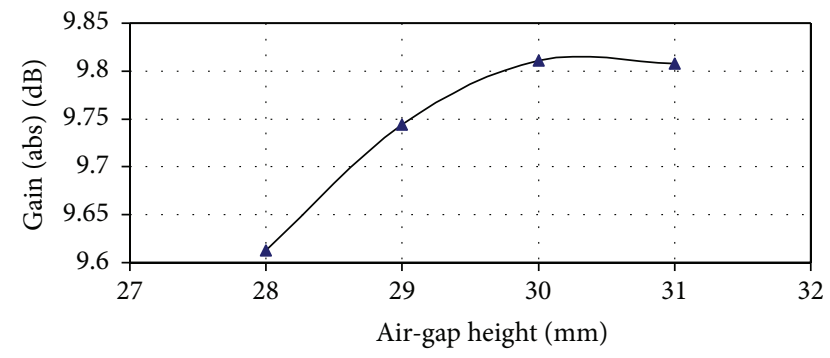

FIgURE 15: Gain versus air-gap height at $5.8 \mathrm{GHz}$.

the Roger 4003 substrate with the thickness of $0.8 \mathrm{~mm}$. The circular polarization is obtained by trimming opposite corners of a square patch [27] and exciting at the feed point as in Figure 13(a). A common measure for the quality of the achieved circular polarization is the axial ratio $\mathrm{AR}_{\mathrm{RA}}=$ $E_{\max } / E_{\min }=-0.08 \mathrm{~dB}$. This antenna gain is $6.5 \mathrm{dBi}$ and reflection coefficient $S_{11}$ at $5.8 \mathrm{GHz}$ is $-20 \mathrm{~dB}$ and $-15 \mathrm{~dB}$ in simulation and measurement, respectively.

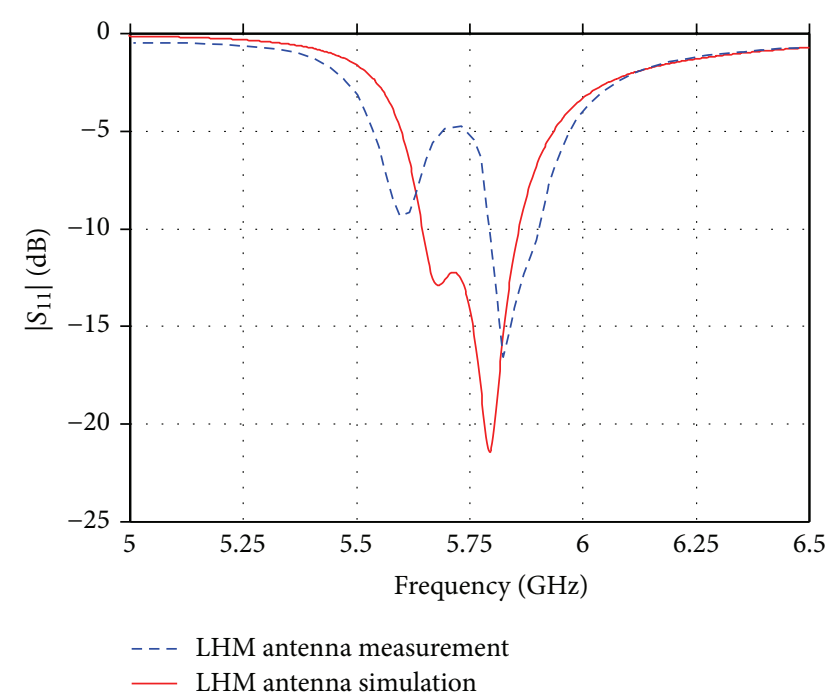

FIGURE 16: Reflection coefficients of FL-LHM antenna with $h=$ $30 \mathrm{~mm}$ in simulation and measurement.

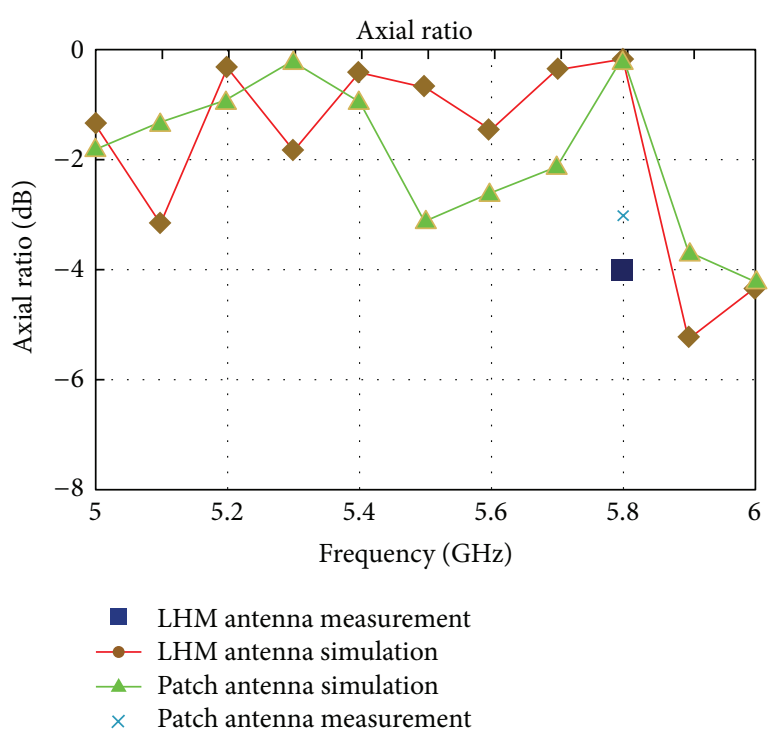

Figure 17: Axial ratio of reference antenna and FL-LHM antenna. 


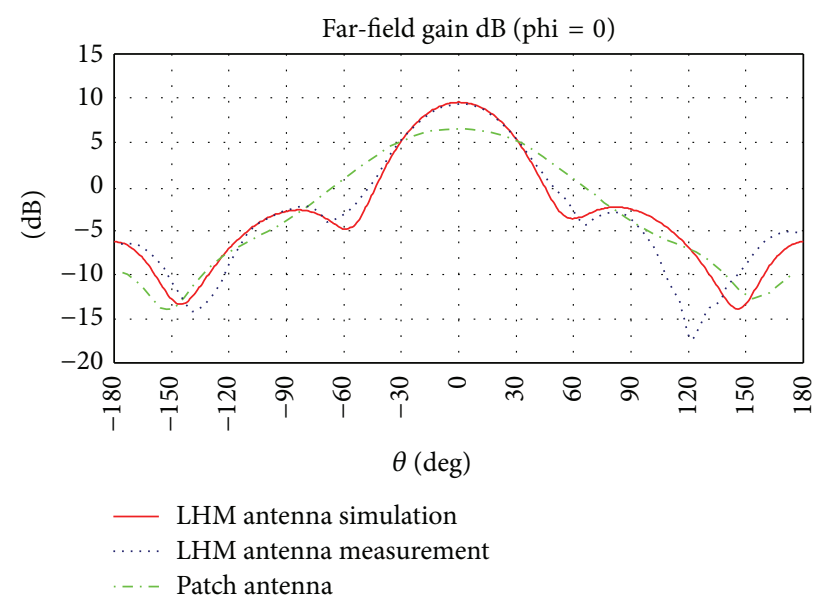

FIGURE 18: Radiation pattern of fabricated FL-LHM antenna in simulation and measurement.

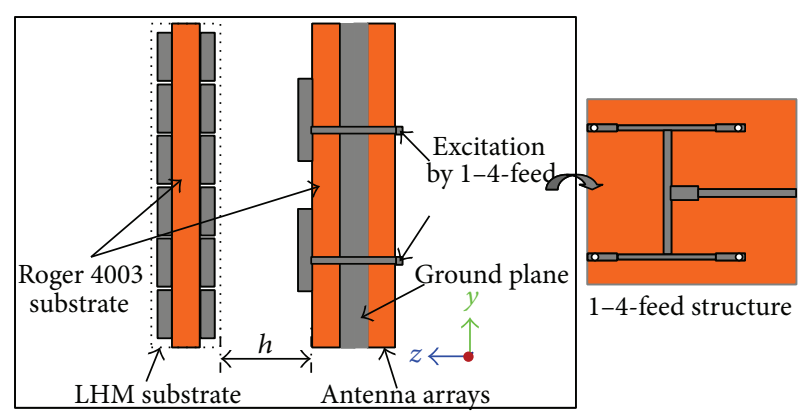

FIGURE 19: Structure of FL-LHM antenna arrays using 1-4-feed structure.

Our study shows that FL-LHM antenna is well matched with air-gap heights from 28 to $31 \mathrm{~mm}$ (Figure 14). In this case, dimensions of fabricated FL-LHM substrate are defined by $L_{x}=L_{y}=46 \mathrm{~mm}$. Figure 15 illustrates the FL-LHM antenna gain versus air-gap height at the frequency of $5.8 \mathrm{GHz}$. The chosen air-gap height of $30 \mathrm{~mm}$ gives the good circular polarization with $\mathrm{AR}_{\mathrm{LHM}-\mathrm{A}}=-0.12 \mathrm{~dB}$ and highest gain (Figure 15) while $S_{11}<-20 \mathrm{~dB}$ at $5.8 \mathrm{GHz}$. The simulated gain is increased from $6.6 \mathrm{dBi}$ to $9.8 \mathrm{dBi}$ by using this FL-LHM layer; the measured gain is $9.5 \mathrm{dBi}$ (Figure 16). The reflection coefficients, axial ratios, and radiation pattern of FL-LHM antenna are shown in Figures 17 and 18.

3.2. Antenna Arrays Gain Enhancement. From the patch antenna designed in Section 3.1, an array of $2 \times 2$ patch antennas is created using the 1-4-feed structure as in Figure 19. The antenna arrays gain is $12.7 \mathrm{dBi}$ and $12.1 \mathrm{dBi}$ in simulation and measurement, respectively (see Figure 20).

When this antenna is covered by FL-LHM substrate with the air-gap height $h=30 \mathrm{~mm}$, the $S_{11}$ is $-12 \mathrm{~dB}$ and $-14 \mathrm{~dB}$

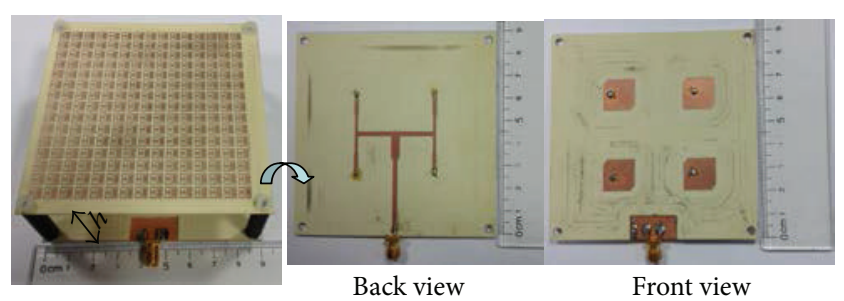

FIgURE 20: Prototype of FL-LHM antenna arrays with dimensions of $90 \times 90 \times 30 \mathrm{~mm}^{3}$.

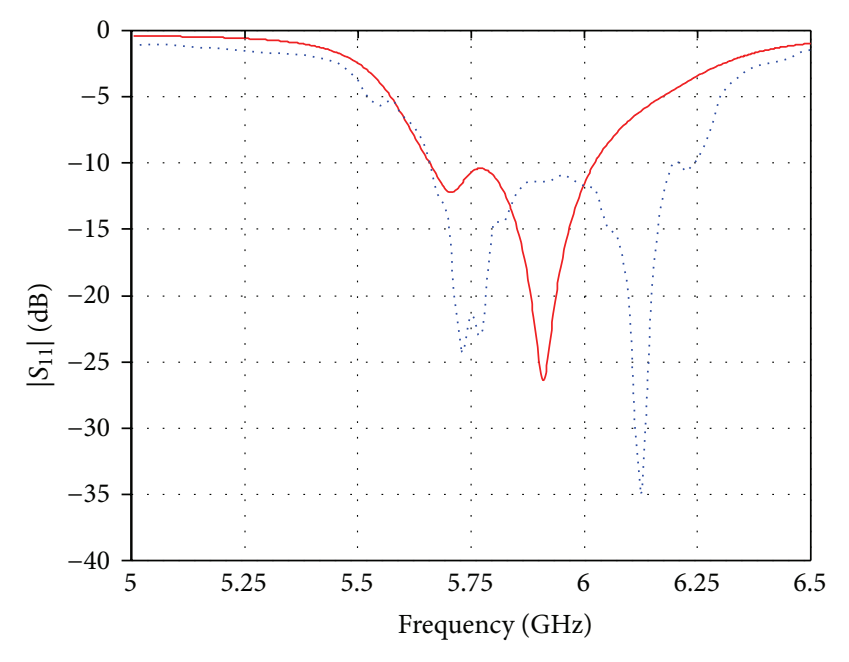

$\ldots S_{11}$ measurement
$-S_{11}$ simulation

FIGURE 21: Reflection coefficients of FL-LHM antenna arrays with $h=30 \mathrm{~mm}$ in simulation and measurement.

(Figure 21) while the gain is improved to $15.3 \mathrm{dBi}$ and $15.4 \mathrm{dBi}$ (Figure 22) in simulation and measurement, respectively.

3.3. Beam Steering Antenna Gain Enhancement. The reference antenna is used as a beam steering antenna using two passive patches at the right side and the left side of the active patch (driven element) in $x$ direction (Figure 23(a)), according to [28]. The active patch is excited by RF source; two patches passive at the right side (patch 2) and at the left side (patch 3) are loaded by the reactive elements $C 2$ and C3, respectively. The mutual couplings between three patches are proportional to the distance $d s$ between them [29]. The current magnitude on the passive radiator is larger when $d s$ is smaller so that the gain will be increased.

The phases shifted between antenna elements are turned by changing the reactive load. We denote by $I_{1}$ the current on the active patch; $I_{2}$ and $I_{3}$ are the induced currents on passive patches $P 2$ and $P 3$, respectively. The array factor is given by [28]

$$
\mathrm{AF}=\sum_{i=1}^{3}\left|\frac{I_{i}}{I_{1}}\right| e^{j\left(k * d_{x} * \sin \theta+\operatorname{ang}\left(I_{i} / I_{1}\right)\right)}
$$




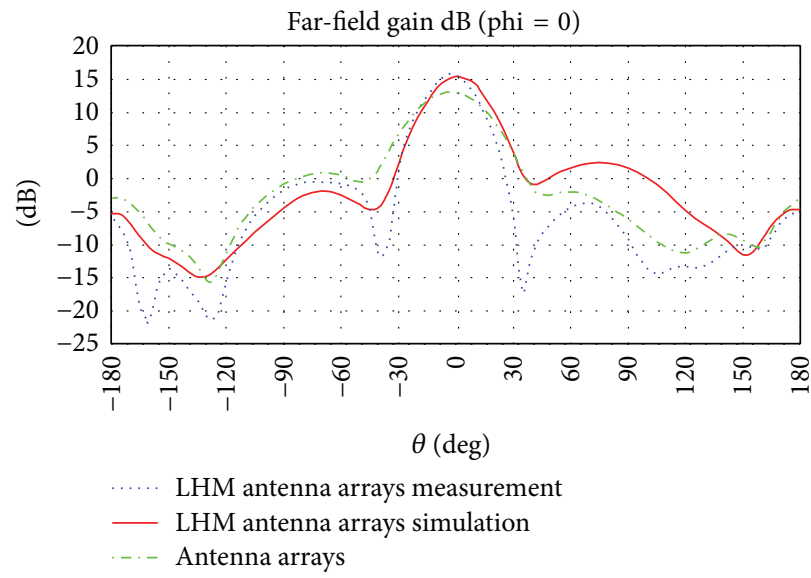

FIGURE 22: Radiation pattern of fabricated FL-LHM antenna arrays in simulation and measurement.

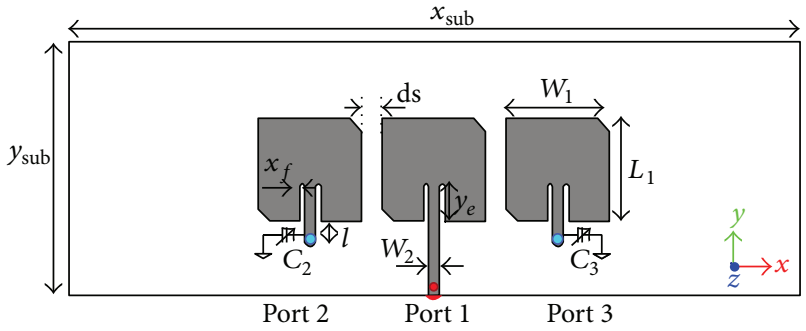

(a)

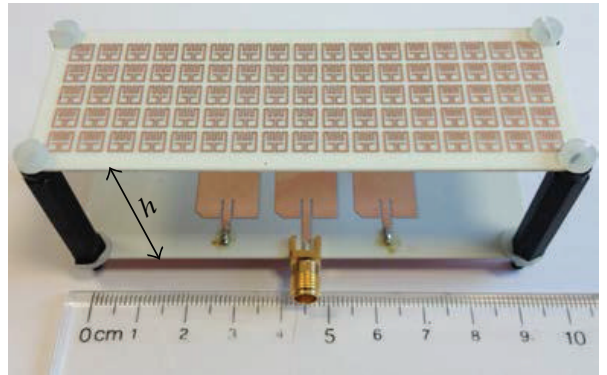

(b)

FIGURE 23: Structure of FL-LHM beam steering antenna: (a) beam steering reference antenna; (b) FL-LHM beam steering antenna with $h=30 \mathrm{~mm}$.

TABLE 3: Parameters of LHM beam steering antenna at $5.8 \mathrm{GHz}$ as in Figure 23.

\begin{tabular}{lc}
\hline Symbol & Value \\
\hline$W_{1}=L_{1}$ & $14(\mathrm{~mm})$ \\
$\mathrm{ds}$ & $3(\mathrm{~mm})($ gap between two patches) \\
$y_{e}$ & $5(\mathrm{~mm})$ \\
$x_{f}$ & $0.5(\mathrm{~mm})$ \\
$W_{2}$ & $1.7(\mathrm{~mm})$ \\
$l$ & $2(\mathrm{~mm})$ \\
$h$ & $30(\mathrm{~mm})$ \\
$x_{\text {sub }}$ & $30(\mathrm{~mm})($ substrate Roger 4003$)$ \\
$y_{\text {sub }}$ & $90(\mathrm{~mm})$ \\
\hline
\end{tabular}

The steering of reference antenna is described in the following three cases (Figures 24 and 25).

(i) Case 1: $C 2=C 3=0.2 \mathrm{pF}$; the AF is maximum; the main lobe is located at theta of $0^{\circ}$. Case 1 is noted in red color in all figures. (ii) Case 2: $C 2=2 \mathrm{pF}$ and $C 3=0.2 \mathrm{pF}$; the main lobe is steered at theta of $20^{\circ}$. Case 2 is presented in green color.

(iii) Case 3: inversely, if $C 2=0.2 \mathrm{pF}$ and $C 3=2 \mathrm{pF}$, the main lobe is steered at theta of $-20^{\circ}$. Case 3 is represented in blue color.

Figures 24 and 25 represent the $S_{11}$ and radiation pattern of beam steering reference antenna in simulation and measurement, respectively.

Our study found that the beam steering reference antenna is always adaptive in three cases at $5.8 \mathrm{GHz}\left(S_{11 \text { simulation }}=\right.$ $-15 \mathrm{~dB}, S_{11 \text { measurement }}=-20 \mathrm{~dB}$ ); the peak of $S_{11}$ is shifted at the higher frequency in case 2 and case 3 . The gain of RA is around $8.2 \mathrm{dBi} / 8.3 \mathrm{dBi}$ in measurement and simulations for case 1 . The gain reduces to $7.7 \mathrm{dBi}$ in simulation and to $7.5 \mathrm{dBi}$ in measurement for cases 2 and 3 (Table 3).

The enhancement gain will be obtained when beam steering antenna is covered by the FL-LHM substrate as in Figure 23(b). The FL-LHM beam steering antenna is well matched at the range of 5.75-5.87 GHz (Figure 26) that covers the DSRC standard. However, the steering angles are reduced 


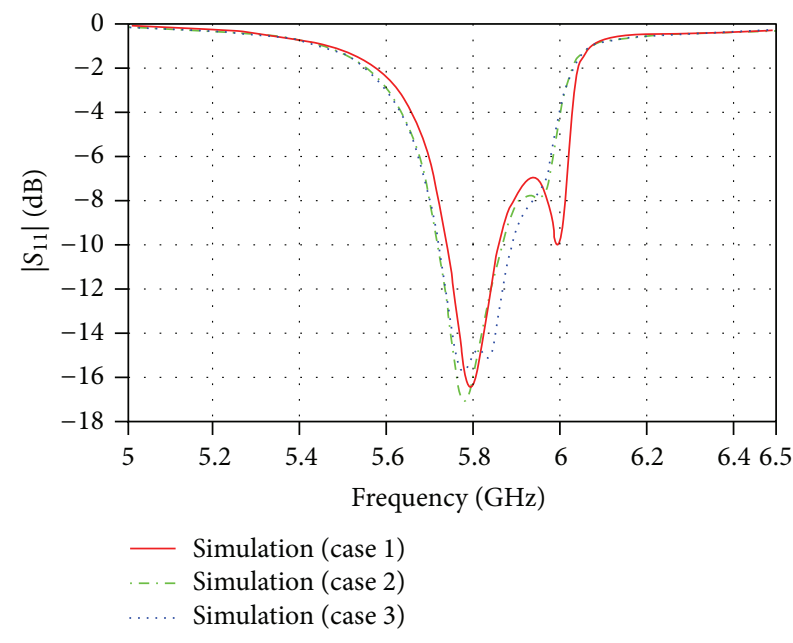

(a)

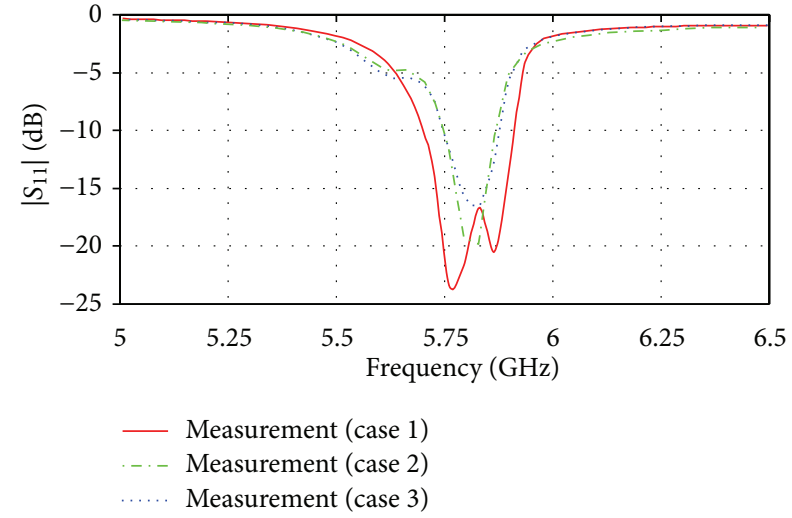

(b)

FIGURE 24: (a) Reflection coefficients of beam steering reference antenna in simulation. (b) Reflection coefficients of beam steering reference antenna in measurement.

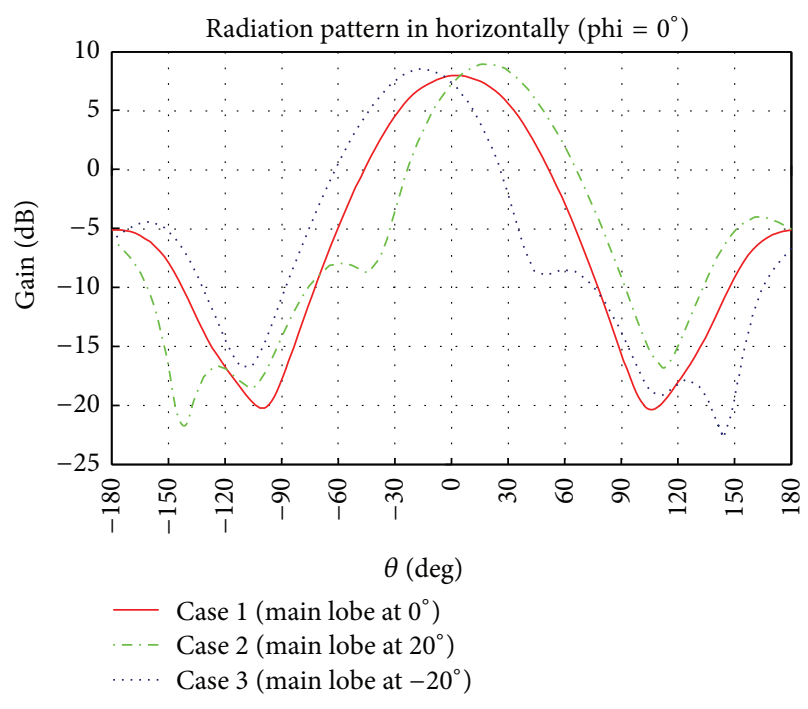

(a)

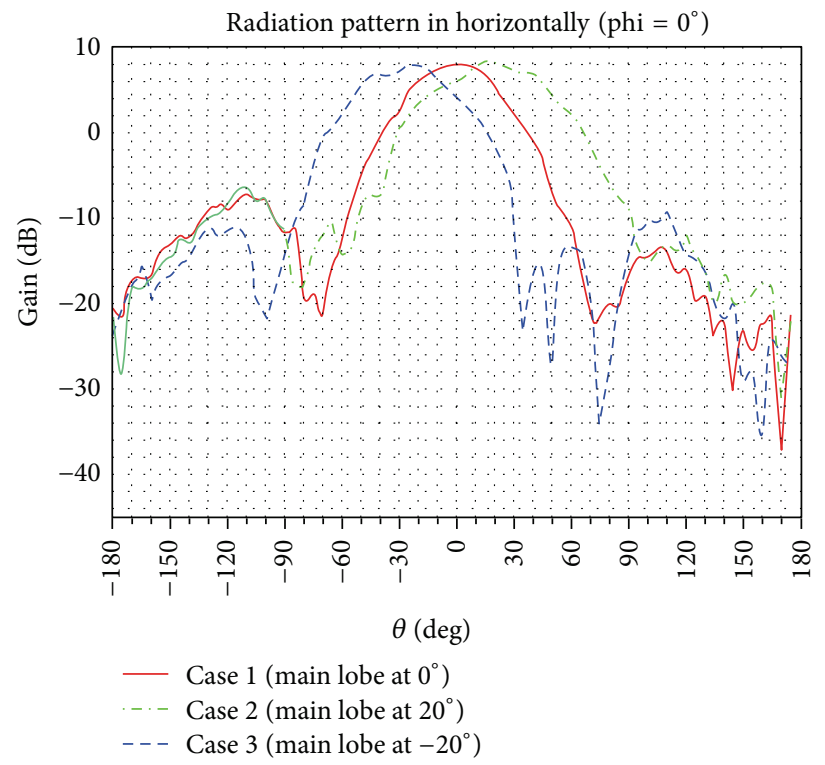

(b)

Figure 25: (a) Radiation pattern of beam steering RA in simulation. Beam steering horizontally; steering angles: $-20^{\circ}$ (case 2), $0^{\circ}$ (case 1), and $20^{\circ}$ (case 3). (b) Radiation pattern of beam steering RA in measurement. Beam steering horizontally; steering angles: $-20^{\circ}$ (case 2 ), $0^{\circ}$ (case 1 ), and $20^{\circ}$ (case 3 ).

to $\pm 10^{\circ}$ instead of $\pm 20^{\circ}$ because of FL-LHM effect according to Snell's law when the waves propagate through FL-LHM substrate.

The reflection coefficient $S_{11}$ and radiation pattern of FLLHM beam steering antenna in three cases are shown in Figures 26 and 27. This FL-LHM antenna has dimensions of $30 \times 90 \times 30 \mathrm{~mm}^{3}$.
In simulation, the gain of beam steering antenna is improved from $8.2 \mathrm{dBi}$ to $12 \mathrm{dBi}$ for case 1 and from $7.7 \mathrm{dBi}$ to $11 \mathrm{dBi}$ for cases 2 and 3 (Figure 27(a)).

In measurement, the gain obtained is $11.6 \mathrm{dBi}$ for case 1 and $10 \mathrm{dBi}$ for cases 2 and 3 (Figure 27(b)). The difference of FL-LHM antenna gain between case 1 and case 2/case 3 is caused by the limited condition of the FL-LHM substrate 


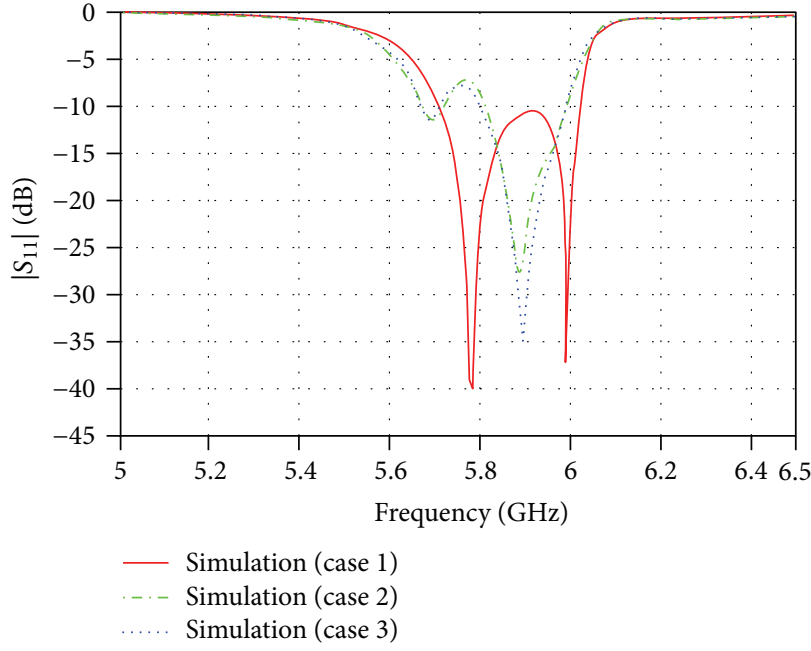

(a)

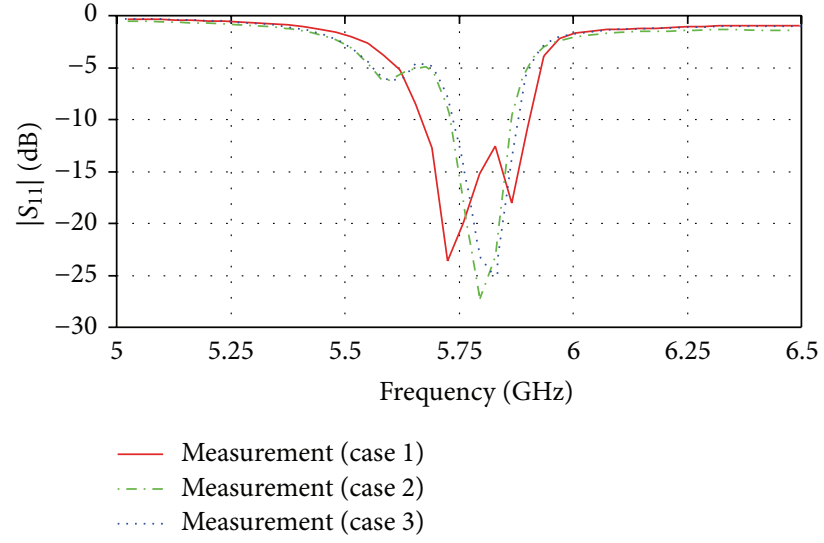

(b)

FIGURE 26: (a) Reflection coefficients of FL-LHM beam steering antenna with $h=30 \mathrm{~mm}$ in simulation. (b) Reflection coefficients of FL-LHM beam steering antenna with $h=30 \mathrm{~mm}$ in measurement.

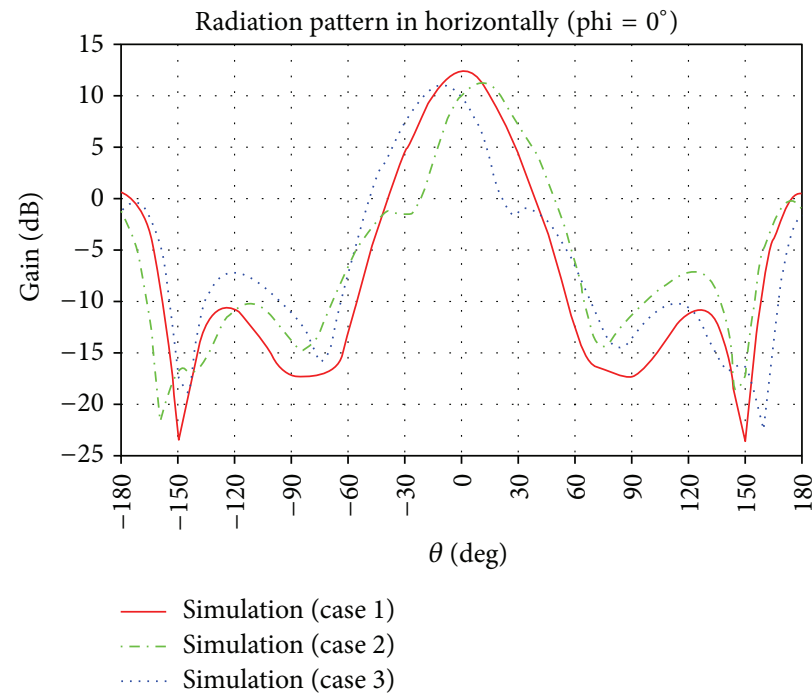

(a)

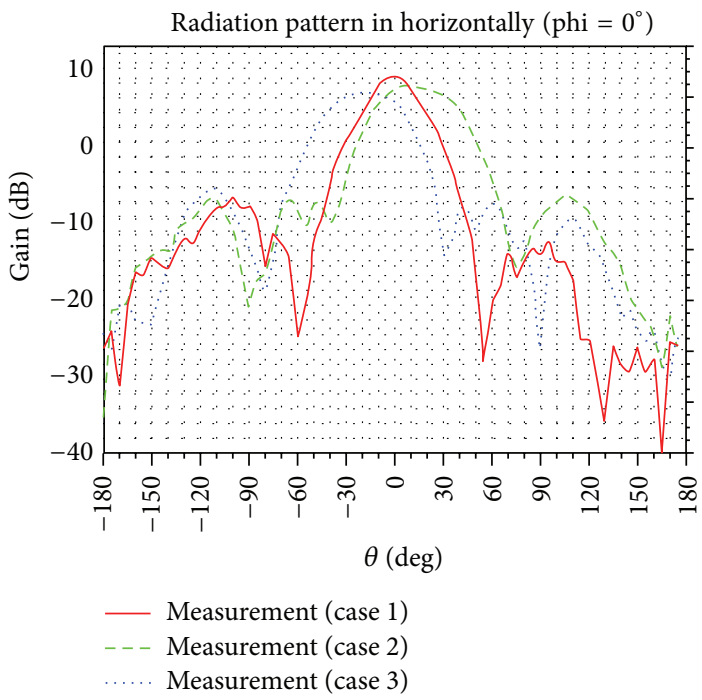

(b)

FIGURE 27: (a) Radiation pattern of FL-LHM beam steering antenna with $h=30 \mathrm{~mm}$ in simulation. Beam steering horizontally; steering angles: $-10^{\circ}$ (case 2), $0^{\circ}$ (case 1), and $10^{\circ}$ (case 3). (b) Radiation pattern of FL-LHM beam steering antenna with $h=30 \mathrm{~mm}$ in measurement. Beam steering horizontally; steering angles: $-10^{\circ}$ (case 2 ), $0^{\circ}$ (case 1 ), and $10^{\circ}$ (case 3 ).

that is analyzed in Section 2.2 as well as the effect of capacitor loaded in passive patches.

Table 4 resumes the simulation and measurement results of FL-LHM beam steering antenna in three cases.

\section{Conclusion}

In this paper, a new planar FL-LHM structure is presented. An equivalent circuit is useful for understanding and designing a FL-LHM substrate for an arbitrary operating frequency. In addition, the FL-LHM modeling is created for easy simulation using electromagnetic software and for enhancement antenna gain. In consequence, the new FLLHM substrate is used to increase the gain of three types of low-profile antennas which are the circularly polarized rectangular patch antenna, the antenna arrays, and the beam steering antenna. These three low-profile FL-LHM antennas operate at the frequency according to the DSRC standard for 
TABLE 4: Simulation and measurement results of LHM beam steering antenna.

\begin{tabular}{lcccccc}
\hline \multirow{2}{*}{$f=5.8 \mathrm{GHz}$} & \multicolumn{2}{c}{ CHM beam steering antenna $\left(30 \times 90 \times 30 \mathrm{~mm}^{3}\right)$} \\
& Sim. & Meas. & Sim. & Meas. & Sim. & Meas. \\
\hline$S_{11}(\mathrm{~dB})$ & -20 & -15 & -11 & -27 & -10 & -24 \\
\hline BW $(\mathrm{MHz})$ & 277 & 250 & 179 & 120 & 194 & 140 \\
\hline Peak gain $(\mathrm{dBi})$ & 12 & 11.6 & 11.1 & 10 & 11 & 9.8 \\
\hline$\Delta G(\mathrm{dBi})$ & 3.8 & 3.3 & 3.4 & 2.5 & 3.2 & 2.3 \\
\hline
\end{tabular}

Sim.: simulation.

Meas.: measurement.

BW: bandwidth.

$\Delta G$ : increased gain by using FL-LHM substrate (compared with RA).

ETC free-flow system application. The gains measured are $9.5 \mathrm{dBi}, 15.3 \mathrm{dBi}$, and $11 \mathrm{dBi}$ in measurement. The gain of any $\mathrm{RA}$ is increased up to around $2.5-3 \mathrm{dBi}$ by using this planar FL-LHM substrate. The $S_{11}$ and radiation pattern results in measurement of three FL-LHM antennas are well fit with simulation results.

\section{Conflict of Interests}

The authors declare that there is no conflict of interests regarding the publication of this paper.

\section{Acknowledgments}

The authors wish to thank A. Gachon (IMEP-LAHC) for his help in fabrication and $\mathrm{K}$. Belmkaddem (CEA-LETI) for her help in the measurement of the prototypes A and B.

\section{References}

[1] V. G. Veselago, "The electrodynamics of substances with simultaneously negative values of $\varepsilon$ and $\mu$," Soviet Physics Uspekhi, vol. 10, no. 4, pp. 509-514, 1968.

[2] J. B. Prendry, "Extremely low frequency plasmons in metallic mesostructures," Physical Review Letters, vol. 76, p. 4773, 1996.

[3] J. B. Prendry, A. J. Holden, D. J. Robbins, and W. J. Stewart, "Magnetism from conductors and enhanced nonlinear phenomena," IEEE Transactions on Microwave Theory and Techniques, vol. 47, no. 11, pp. 2075-2084, 1999.

[4] D. R. Smith, D. C. Vier, N. Kroll, and S. Schultz, "Direct calculation of permeability and permittivity for a left-handed metamaterial," Applied Physics Letters, vol. 77, article 2246, no. $14,2000$.

[5] D. R. Smith, W. J. Padilla, D. C. Vier, S. C. Nemat-Nasser, and S. Schultz, "Composite mediu $\mathrm{m}$ with simultaneously negative permeability and permittivity," Physical Review Letters, vol. 84, no. 18, pp. 4184-4187, 2000.

[6] R. W. Ziolkowski, "Design, fabrication, and testing of double negative metamaterials," IEEE Transactions on Antennas and Propagation, vol. 51, no. 7, pp. 1516-1529, 2003.

[7] M. M. I. Saadoun and N. Engheta, "A reciprocal phase shifter using novel pseudochiral or $\omega$ medium," Microwave and Optical Technology Letters, vol. 5, no. 4, pp. 184-188, 1992.
[8] C. R. Simovski, S. A. Tretyakov, A. A. Sochava, B. Sauviac, F. Mariotte, and T. G. Kharina, "Antenna model for conductive omega particles," Journal of Electromagnetic Waves and Applications, vol. 11, no. 11, pp. 1509-1530, 1997.

[9] C. R. Simovski, "Plane-wave reflection and transmission by grids of conducting $\Omega$-particles and dispersion of $\Omega$ electromagnetic crystals," AEU-International Journal of Electronics and Communications, vol. 57, no. 5, pp. 358-364, 2003.

[10] É. Lheurette, G. Houzet, J. Carbonell, F. Zhang, O. Vanbésien, and D. Lippens, "Omega-type balanced composite negative refractive index materials," IEEE Transactions on Antennas and Propagation, vol. 56, no. 11, pp. 3462-3469, 2008.

[11] H. Chen, L. Ran, J. Huangfu et al., "Left-handed materials composed of only S-shaped resonators," Physical Review E, vol. 70, Article ID 057605, 2004.

[12] D. R. Smith and J. B. Pendry, "Homogenization of metamaterials by field averaging," Journal of the Optical Society of America B, vol. 23, no. 3, pp. 391-403, 2006.

[13] A. Ramakrishna and J. Pendry, "Non-linear effects in negative magnetive mata-materials," Physical Review, vol. 4, 2006.

[14] R. Liu, T. J. Cui, D. Huang, B. Zhao, and D. R. Smith, “Description and explanation of electromagnetic behaviors in artificial metamaterials based on effective medium theory," Physical Review E-Statistical, Nonlinear, and Soft Matter Physics, vol. 76, Article ID 026606, 2007.

[15] D. R. Smith, J. Gollub, J. J. Mock, W. J. Padilla, and D. Schurig, "Calculation and measurement of bianisotropy in a split ring resonator metamaterial," Journal of Applied Physics, vol. 100, no. 2, Article ID 024507, 2006.

[16] X. Chen, T. M. Grzegorczyk, B.-I. Wu, J. Pacheco Jr., and J. A. Kong, "Robust method to retrieve the constitutive effective parameters of metamaterials," Physical Review E-Statistical, Nonlinear, and Soft Matter Physics, vol. 70, Article ID 016608, 2004.

[17] Y. H. Liu and X. P. Zhao, "Investigation of anisotropic negative permeability medium cover for patch antenna," IET Microwaves, Antennas and Propagation, vol. 2, no. 7, pp. 737744, 2008.

[18] T. Zwick, A. Chandrasekhar, C. W. Baks, U. R. Pfeiffer, S. Brebels, and B. P. Gaucher, "Determination of the complex permittivity of packaging materials at millimeter-wave frequencies," IEEE Transactions on Microwave Theory and Techniques, vol. 54, no. 3, pp. 1001-1009, 2006.

[19] P. Markos and C. M. Soukoulis, "Left-handed materials," Physical Review B, vol. 65, Article ID 033401, 2002.

[20] D. McGinnis, "PBAR NOTE 585 Measurement of Ralative permittivity and Permeability using Two Port S-parameter technique," April 1998, http://lss.fnal.gov/archive/pbarnote/ fermilab-pbar-note-585.pdf.

[21] M. T. Le, Q. C. Nguyen, T. P. Vuong, and C. Defay, "New metamaterial structure for the design of a high gain antenna at $5.8 \mathrm{GHz}$, in Proceedings of the IEEE International Conference on Wireless Information Technology and Systems (ICWITS '12), pp. 1-4, Maui, Hawaii, USA, November 2012.

[22] M. T. Le, Q. C. Nguyen, T. T. T. Vu, and T. P. Vuong, "Design of an directive antenna for "free-flow" system application," in Proceedings of the IEEE Conference of Advanced Technologies for Communication, August 2011.

[23] K. Thales, Global Specification for Short Range Communication, Kapsch, Thales, 2003.

[24] CEN, “DIN EN12253," 2002. 
[25] CEN, “NF EN ISO 14906," AFNOR, 2005.

[26] T. J. Cui, "A symmetrical circuit model describing all kinds of circuit metamaterials," Progress in Electromagnetics Research B, vol. 5, pp. 63-76, 2008.

[27] A. Balanis, Antenna Theory Analysis and Design, John Wiley \& Sons, 3rd edition, 2005.

[28] Y. Yusuf and X. Gong, "A low-cost patch antenna phased array with analog beam steering using mutual coupling and reactive loading," IEEE Antennas and Wireless Propagation Letters, vol. 7, pp. 81-84, 2008.

[29] N. G. Alexopoulos and I. E. Rana, "Mutual impedance computation between printed dipoles," IEEE Transactions on Antennas and Propagation, vol. 29, no. 1, pp. 106-111, 1981. 

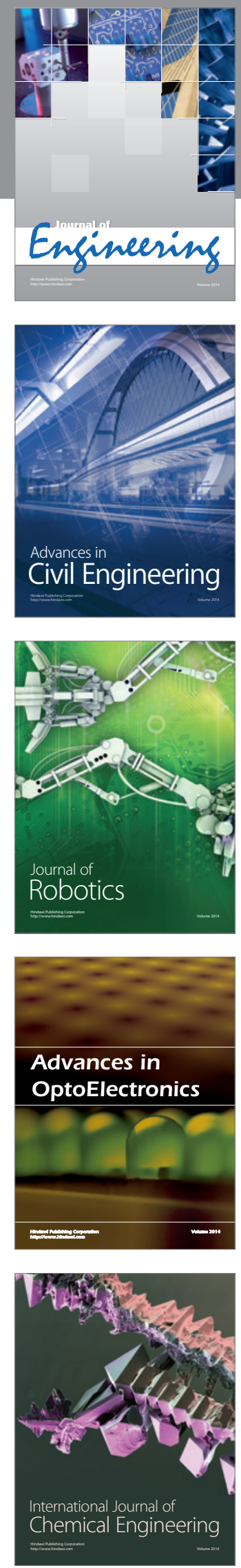

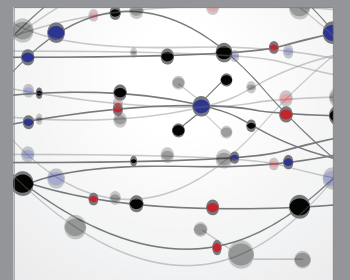

The Scientific World Journal
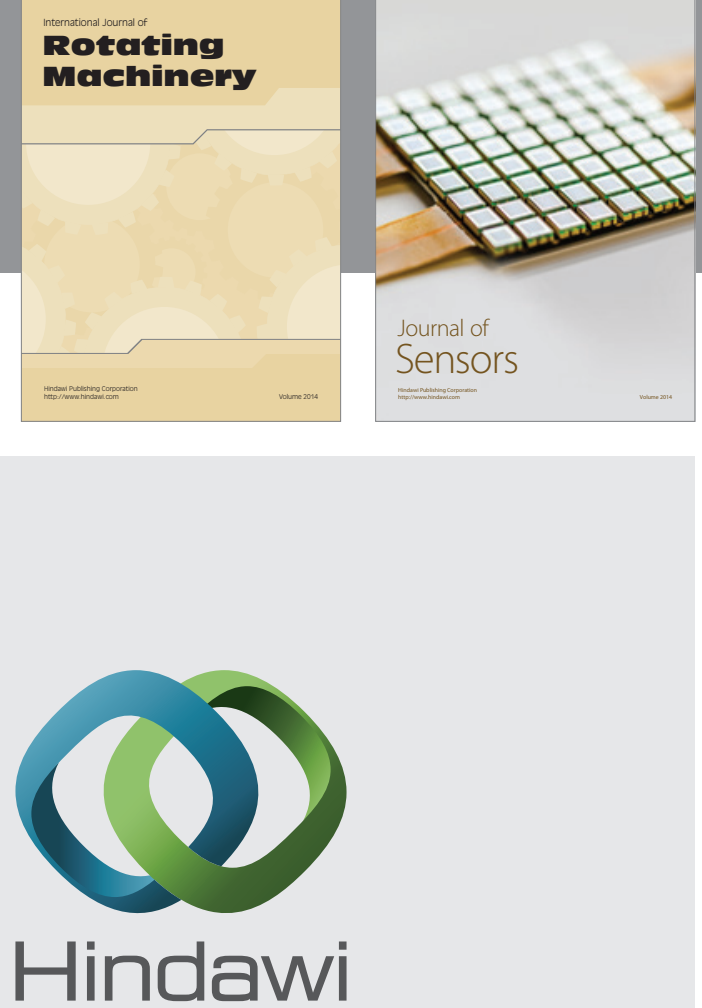

Submit your manuscripts at http://www.hindawi.com
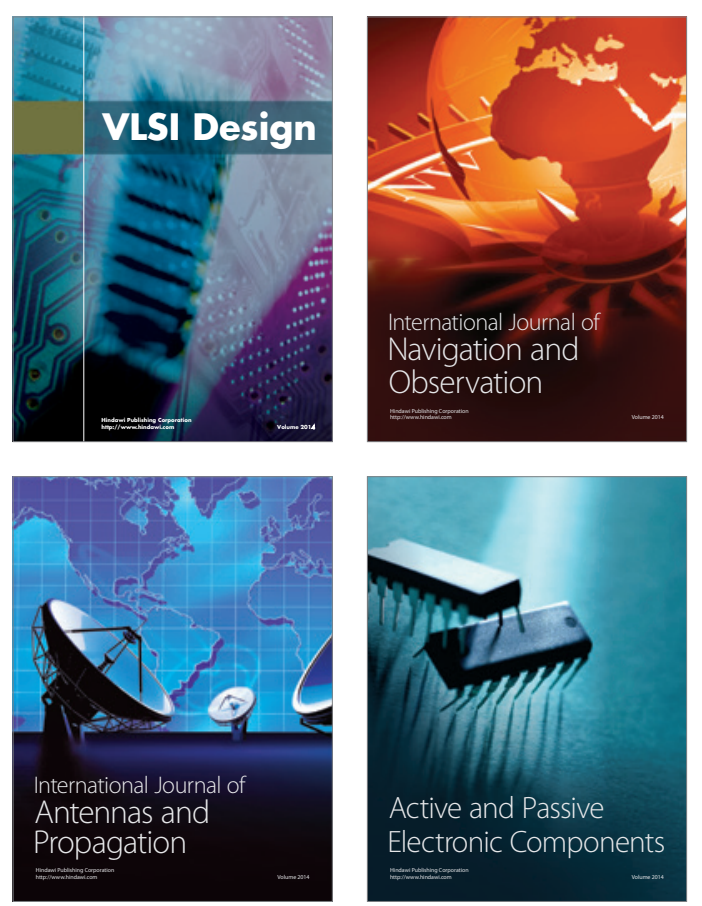
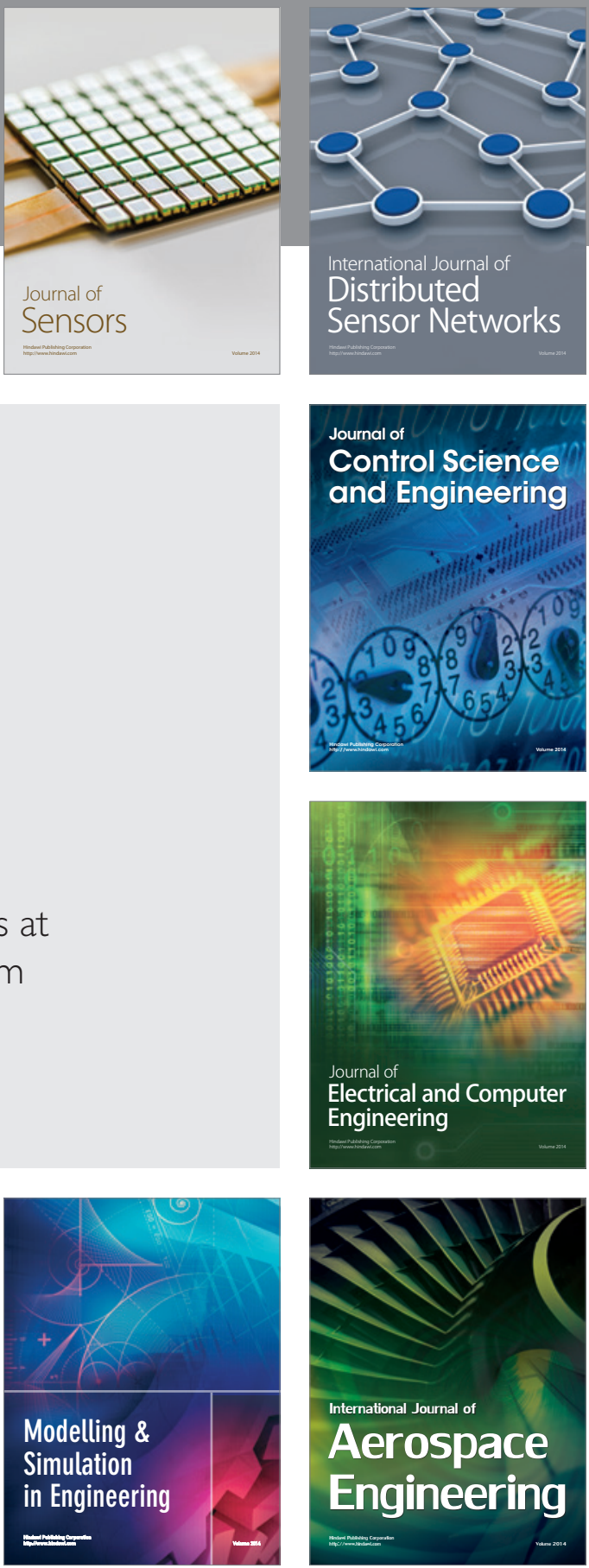

Journal of

Control Science

and Engineering
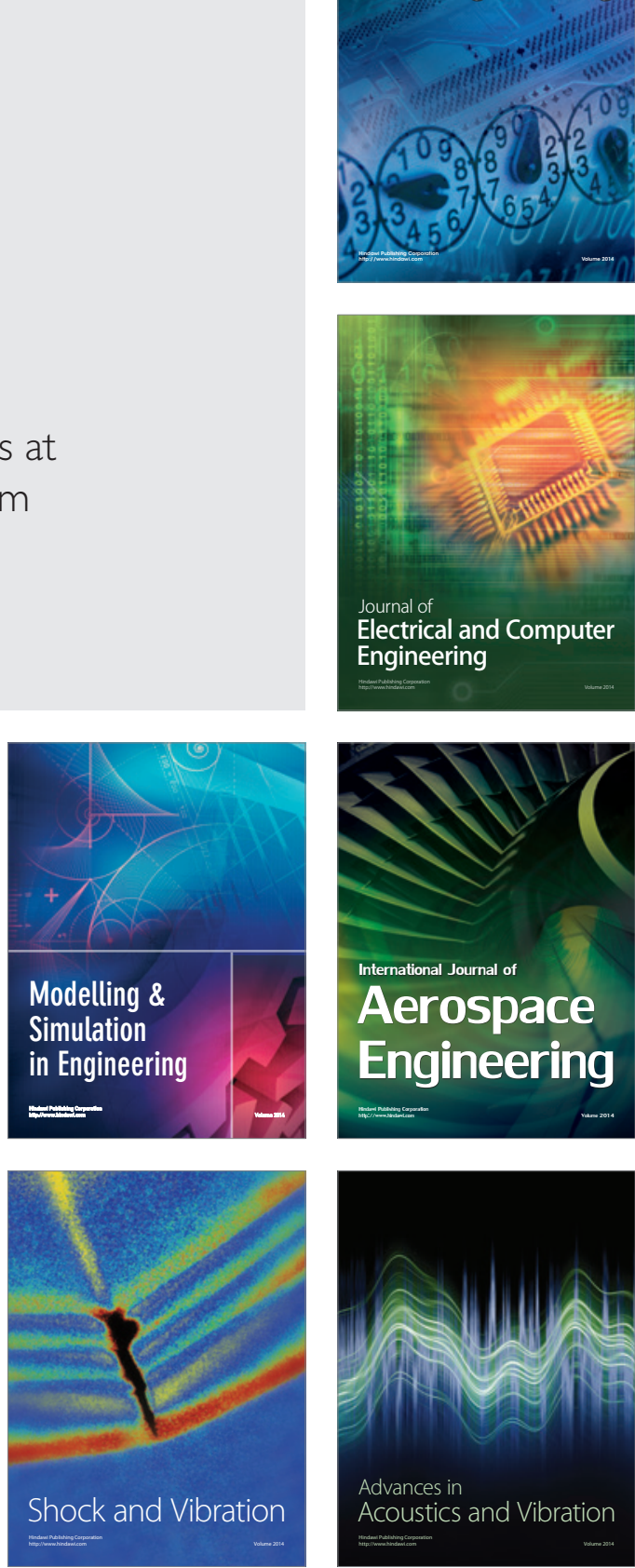